\title{
Ordinary state-based peridynamics for plastic deformation according to von Mises yield criteria with isotropic hardening
}

\author{
Erdogan Madenci* and Selda Oterkus \\ Department of Aerospace and Mechanical Engineering, The University of Arizona, Tucson, AZ \\ 85721, United States
}

\begin{abstract}
This study presents the ordinary state-based peridynamic constitutive relations for plastic deformation based on von Mises yield criteria with isotropic hardening. The peridynamic force density-stretch relations concerning elastic deformation are augmented with increments of force density and stretch for plastic deformation. The expressions for the yield function and the rule of incremental plastic stretch are derived in terms of the horizon, force density, shear modulus, and hardening parameter of the material. The yield surface is constructed based on the relationship between the effective stress and equivalent plastic stretch. The validity of peridynamic predictions is established by considering benchmark solutions concerning a plate under tension, a plate with a hole and a crack also under tension.
\end{abstract}

Keywords: peridynamics, plasticity, isotropic, hardening, failure

\section{Introduction}

Structural metals exhibit plastic deformation when loaded beyond their elastic limit. In the absence of cracks, their behavior is well understood from a computational point of view within the classical continuum mechanics. Fracture of such components is often preceded by an extensive plastic deformation. The traditional approaches to predict failure usually employ concepts from linear elastic fracture mechanics (LEFM), which is conceptually limited to materials exhibiting brittle behavior. Therefore, the applicability of fracture toughness as defined by LEFM becomes questionable in the presence of plastic deformation. Furthermore, the assumption of a sharp crack tip in LEFM may no longer be valid due to the presence of plastic deformation. Also, unlike elastic fracture, ductile fracture is inherently a path-dependent process involving irreversible energy dissipation by yielding and fracturing of materials.

Peridynamics (PD), introduced by Silling (2000), is a reformulation of the classical continuum mechanics equations that introduces an internal length scale that is lacking in the classical form of the equations. Peridynamics is based on integro-differential equations as opposed to the partial differential equations of classical continuum mechanics. It is extremely suitable for failure analysis of structures because it allows cracks to grow naturally without resorting to external crack growth laws. An extensive literature survey on peridynamics is given by Madenci and Oterkus (2014). Peridynamics is not limited to linear elastic material behavior. As part of "nonordinary" state-based peridynamics, Taylor (2008) and Foster et al. (2010) considered viscoplastic material behavior. Also, Foster et al. (2011) proposed critical energy density as an alternative critical parameter for such material behavior. However, "nonordinary" state-based models employ constitutive relations

\footnotetext{
* Corresponding author. Tel.: +1 5206216113.

E-mail address: madenci@arizona.edu (E. Madenci).
} 
that are non-native to PD theory. It is prone to oscillations in the regions of steep gradients such as the crack tip. The source of such oscillations is due to the inadequate approximation in the force density vector. Breitenfeld et al. (2014) presented various non-mathematical techniques to reduce these oscillations. Free of such oscillations, Mitchell (2011) presented an ordinary state-based PD model for ideal plastic deformation of materials in the absence of crack propagation.

This study presents an ordinary state-based PD plasticity model in accordance with von Mises yield criteria and isotropic hardening. Also, it presents a peridynamic J-integral based damage criteria to predict crack propagation. Furthermore, it includes an innovative approach to impose nonlocal boundary conditions. The peridynamic predictions concern equivalent plastic stretch and effective stress in a plate with a hole and a crack along with J-integral calculations.

\section{Peridynamic equation of motion}

The peridynamic equation of motion introduced by Silling (2000) and later generalized by Silling et al. (2007) is a nonlinear integro-differential equation in time and space in the form

$$
\rho(\mathbf{x})^{\cdot *}, \quad, \quad \int_{H}\left(\mathbf{t}\left(\mathbf{u}^{\prime}-\mathbf{u}, \mathbf{x}^{\prime}-\mathbf{x}, t\right)-\mathbf{t}^{\prime}\left(\mathbf{u}^{\prime}-\mathbf{u}, \mathbf{x}^{\prime}-\mathbf{x}, t\right)\right) d H+\mathbf{b}(\mathbf{x}, t),
$$

which can be discretized as

$$
\rho_{(k)} \quad . . . \quad \stackrel{N}{\sum_{j=1}^{N}}\left[\mathbf{t}_{(k)(j)}\left(\mathbf{u}_{(j)}-\mathbf{u}_{(k)}, \mathbf{x}_{(j)}-\mathbf{x}_{(k)}, t\right)-\mathbf{t}_{(j)(k)}\left(\mathbf{u}_{(k)}-\mathbf{u}_{(j)}, \mathbf{x}_{(k)}-\mathbf{x}_{(j)}, t\right)\right] V_{(j)}+\mathbf{b}_{(k)},
$$

in which each material point is identified by its coordinates, $\mathbf{x}_{(k)}$, and is associated with an incremental volume, $V_{(k)}$, and a mass density, $\rho\left(\mathbf{x}_{(k)}\right)$. With respect to a Cartesian coordinate system, the material point $\mathbf{x}_{(k)}$ experiences displacement, $\mathbf{u}_{(k)}$, and its location is described by the position vector $\mathbf{y}_{(k)}$ in the deformed state. The displacement and body load vectors at material point $\mathbf{x}_{(k)}$ are represented by $\mathbf{u}_{(k)}\left(\mathbf{x}_{(k)}, t\right)$ and $\mathbf{b}_{(k)}\left(\mathbf{x}_{(k)}, t\right)$, respectively. The family of material point $\mathbf{x}_{(k)}$ is denoted by $H_{\mathbf{x}_{(k)}}$, shown in Fig. 1. Similarly, material point $\mathbf{x}_{(j)}$ interacts with material points in its own family, $H_{\mathbf{x}_{(j)}}$.

As illustrated in Fig. 2, the material point $\mathbf{x}_{(k)}$ interacts with its family of material points, $H_{\mathbf{x}_{(k)}}$, and it is influenced by the collective deformation of all these material points, thus resulting in a force density vector, $\mathbf{t}_{(k)(j)}$, acting at material point $\mathbf{x}_{(k)}$. It can be viewed as the force exerted by material point $\mathbf{x}_{(j)}$. Similarly, material point $\mathbf{x}_{(j)}$ is influenced by deformation of the material points, $H_{\mathbf{x}_{(j)}}$, in its own family.

The integrand in Eq. (1a) does not contain any spatial derivatives of displacements. Thus, it is valid everywhere whether or not displacement discontinuities exist in the material. The region $H$ defining the range of material point $\mathbf{x}$ is specified by $\delta$, referred to as the "horizon." Also, 
the material points within a distance $\delta$ of $\mathbf{x}$ are called the family of $\mathbf{x}, H_{\mathbf{x}}$. The locality of interactions depends on the horizon, and the interactions become more local with a decreasing horizon, $\delta$. Hence, the classical theory of elasticity can be considered a limiting case of the peridynamic theory as the horizon approaches zero (Silling and Lehoucq, 2008).

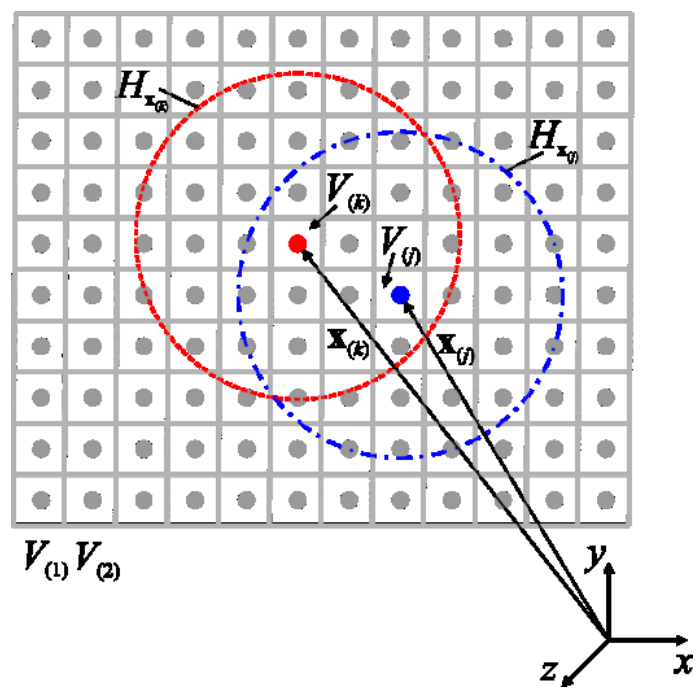

Fig. 1. Peridynamic material points and interaction of points at $\mathbf{x}_{(k)}$ and $\mathbf{x}_{(j)}$.

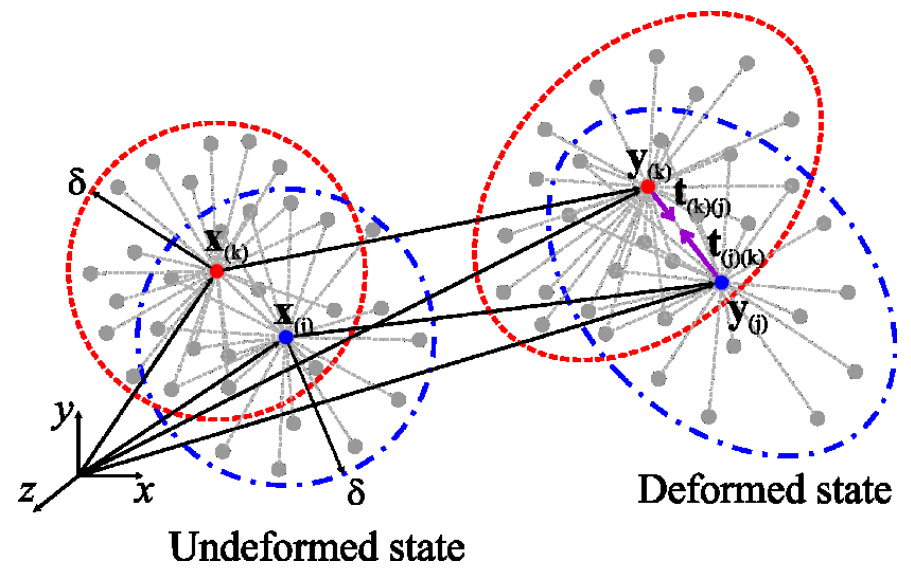

Fig. 2. Peridynamic material points $\mathbf{x}$ and $\mathbf{x}^{\prime}$ influenced by the collective deformation of others in their families.

\section{Peridynamic strain energy density}

For an elastic and isotropic material, the PD strain energy density in terms of deformation at a material point $\mathbf{x}_{(k)}$ can be expressed as (Madenci and Oterkus, 2014) 


$$
W_{(k)}=W_{(k)}^{\kappa}+W_{(k)}^{\mu},
$$

where

$$
W_{(k)}^{\kappa}=a_{\kappa} \theta_{(k)}^{2}
$$

and

$$
W_{(k)}^{\mu}=\left\{b \sum_{j=1}^{N} w_{(k)(j)}\left(\left|\mathbf{y}_{(j)}-\mathbf{y}_{(k)}\right|-\left|\mathbf{x}_{(j)}-\mathbf{x}_{(k)}\right|\right)^{2} V_{(j)}-a_{\mu} \theta_{(k)}^{2}\right\},
$$

with $W_{(k)}^{\kappa}$ and $W_{(k)}^{\mu}$ representing the dilatational and distortional parts of the strain energy density and $a_{\kappa}, a_{\mu}, b$, and $d$ the material parameters. The dilatation $\theta_{(k)}$ can be expressed in discrete form as

$$
\theta_{(k)}=d \sum_{j=1}^{N} w_{(k)(j)}\left(\left|\mathbf{y}_{(j)}-\mathbf{y}_{(k)}\right|-\left|\mathbf{x}_{(j)}-\mathbf{x}_{(k)}\right|\right) \Lambda_{(k)(j)} V_{(j)},
$$

with

$$
\Lambda_{(k)(j)}=\frac{\mathbf{y}_{(j)}-\mathbf{y}_{(k)}}{\left|\mathbf{y}_{(j)}-\mathbf{y}_{(k)}\right|} \cdot \frac{\mathbf{x}_{(j)}-\mathbf{x}_{(k)}}{\left|\mathbf{x}_{(j)}-\mathbf{x}_{(k)}\right|} .
$$

Madenci and Oterkus (2014) gave, the influence function, $w_{(k)(j)}$, in the form

$$
w_{(k)(j)}=\frac{\delta}{\left|\mathbf{x}_{(j)}-\mathbf{x}_{(k)}\right|} .
$$

They also determined the peridynamic parameters $a_{\kappa}, a_{\mu}, b$, and $d$ in terms of engineering material constants by considering two simple loading conditions: isotropic expansion and simple shear. Their specific expressions are given as

$$
\begin{aligned}
& a_{\kappa}=\frac{1}{2} \kappa, a_{\mu}=\frac{5 \mu}{6}, b=\frac{15 \mu}{2 \pi \delta^{5}}, \text { and } d=\frac{9}{4 \pi \delta^{4}} \text { for (3-D), } \\
& a_{\kappa}=\kappa, a_{\mu}=2 \mu, b=\frac{6 \mu}{\pi h \delta^{4}}, \text { and } d=\frac{2}{\pi h \delta^{3}} \text { for (2-D), } \\
& a_{\kappa}=0, a_{\mu}=0, b=\frac{E}{2 \delta^{3} A}, \text { and } d=\frac{1}{2 \delta^{2} A} \text { for (1-D), }
\end{aligned}
$$


with $\kappa$ and $\mu$ representing the bulk modulus and shear modulus of the material. The parameters $h$ and $A$ represent the thickness and cross-sectional area of the structure, respectively. A

parameter $a$, serving as coefficient of the dilatation term, can be defined as $a=a_{\kappa}-a_{\mu}$. If dilatation is not distinguished as in the case of bond-based peridynamics, the parameter $a$ reveals the constraint condition on the Poisson's ratio associated with the bond-based peridynamics as $\kappa=5 \mu / 3$ or $v=1 / 4$ and $\kappa=2 \mu$ or $v=1 / 3$.

The values of these parameters depend on the horizon size, and the dimension of the analysis. It is worth noting that the PD material parameters are determined for material points whose horizons are completely embedded in the material. In other words, they are only valid for a material point whose horizon is not truncated due to the presence of a boundary surface. Otherwise, they need to be corrected in order to account for the loss of family members within the horizon, as explained in Appendix A.

\section{Force density-stretch relations for elastic deformation}

As derived by Madenci and Oterkus (2014), with this representation of the strain energy density, the force density vector, $\mathbf{t}_{(k)(j)}$, at material point $\mathbf{x}_{(k)}$ can be obtained from

$$
\mathbf{t}_{(k)(j)}\left(\mathbf{u}_{(j)}-\mathbf{u}_{(k)}, \mathbf{x}_{(j)}-\mathbf{x}_{(k)}, t\right)=\frac{1}{V_{(j)}} \frac{\partial W_{(k)}}{\partial\left(\left|\mathbf{y}_{(j)}-\mathbf{y}_{(k)}\right|\right)} \frac{\mathbf{y}_{(j)}-\mathbf{y}_{(k)}}{\left|\mathbf{y}_{(j)}-\mathbf{y}_{(k)}\right|},
$$

which leads to the force density vector in the form

$$
\mathbf{t}_{(k)(j)}=t_{(k)(j)} \frac{\mathbf{y}_{(j)}-\mathbf{y}_{(k)}}{\left|\mathbf{y}_{(j)}-\mathbf{y}_{(k)}\right|},
$$

where

$$
t_{(k)(j)}=\left(a_{\kappa}-a_{\mu}\right) 2 \delta d \frac{\Lambda_{(k)(j)}}{\left|\mathbf{x}_{(j)}-\mathbf{x}_{(k)}\right|} \theta_{(k)}+2 \delta b s_{(k)(j)} .
$$

The stretch, $s_{(k)(j)}$, between material points $\mathbf{x}_{(k)}$ and $\mathbf{x}_{(j)}$ is defined as

$$
S_{(k)(j)}=\frac{\left|\mathbf{y}_{(j)}-\mathbf{y}_{(k)}\right|-\left|\mathbf{x}_{(j)}-\mathbf{x}_{(k)}\right|}{\left|\mathbf{x}_{(j)}-\mathbf{x}_{(k)}\right|} .
$$

The force density vector can be decomposed into its dilatational and distortional parts as

$$
\mathbf{t}_{(k)(j)}=\mathbf{t}_{(k)(j)}^{\kappa}+\mathbf{t}_{(k)(j)}^{\mu},
$$


where

$$
\mathbf{t}_{(k)(j)}^{\kappa}=\frac{2 \delta a_{\kappa} d}{\left|\mathbf{x}_{(j)}-\mathbf{x}_{(k)}\right|} \Lambda_{(k)(j)} \theta_{(k)} \frac{\mathbf{y}_{(j)}-\mathbf{y}_{(k)}}{\left|\mathbf{y}_{(j)}-\mathbf{y}_{(k)}\right|}
$$

and

$$
\mathbf{t}_{(k)(j)}^{\mu}=\left(2 \delta b s_{(k)(j)}-\frac{2 \delta a_{\mu} d}{\left|\mathbf{x}_{(j)}-\mathbf{x}_{(k)}\right|} \Lambda_{(k)(j)} \theta_{(k)}\right) \frac{\mathbf{y}_{(j)}-\mathbf{y}_{(k)}}{\left|\mathbf{y}_{(j)}-\mathbf{y}_{(k)}\right|}
$$

By using Eqs. (8b) and (8c), the dilatation, $\theta_{(k)}$, and stretch, $s_{(k)(j)}$, can be expressed in terms of force density as

$$
\theta_{(k)}=t_{(k)(j)}^{\kappa} \frac{\left|\mathbf{x}_{(j)}-\mathbf{x}_{(k)}\right|}{2 \delta a_{\kappa} d \Lambda_{(k)(j)}}
$$

and

$$
s_{(k)(j)}=\frac{1}{2 \delta b}\left[t_{(k)(j)}^{\mu}\right]+a_{\mu} \frac{d}{b}\left[\frac{\Lambda_{(k)(j)}}{\left|\mathbf{x}_{(j)}-\mathbf{x}_{(k)}\right|} \theta_{(k)}\right]
$$

The total stretch can also be decomposed into its dilatational and distortional parts as

$$
S_{(k)(j)}=S_{(k)(j)}^{\kappa}+S_{(k)(j)}^{\mu},
$$

where

$$
s_{(k)(j)}^{\mu}=\frac{1}{2 \delta b}\left[t_{(k)(j)}^{\mu}\right]
$$

and

$$
s_{(k)(j)}^{\kappa}=\frac{a_{\mu} d}{b}\left[\frac{\Lambda_{(k)(j)}}{\left|\mathbf{x}_{(j)}-\mathbf{x}_{(k)}\right|} \theta_{(k)}\right] \text {. }
$$

Substituting for the influence function $w_{(k)(j)}$ from Eq. (4) and rearranging terms in Eq. (2c), the distortional part of the strain energy density expression can be rewritten in terms of stretch as 


$$
W_{(k)}^{\mu}=b \sum_{j=1}^{N} \delta s_{(k)(j)}^{2}\left|\mathbf{x}_{(j)}-\mathbf{x}_{(k)}\right| V_{(j)}-a_{\mu} \theta_{(k)}^{2}
$$

Similarly, substituting for the stretch $s_{(k)(j)}$ from Eq. (9b), this equation can be expressed in terms of force densities as

$$
W_{(k)}^{\mu}=b \sum_{j=1}^{N} \delta\left(\frac{1}{2 \delta b} t_{(k)(j)}^{\mu}+a_{\mu} \frac{d}{b} \frac{\Lambda_{(k)(j)}}{\left|\mathbf{x}_{(j)}-\mathbf{x}_{(k)}\right|} \theta_{(k)}\right)^{2}\left|\mathbf{x}_{(j)}-\mathbf{x}_{(k)}\right| V_{(j)}-a_{\mu} \theta_{(k)}^{2} .
$$

\section{Peridynamic theory for plastic deformations}

If a body experiences plastic deformation, the stretch depends not only on the final force density vector, but also on the loading history. Because the plastic deformation is an irreversible process, it can only be described by introducing response functions. This ensures irreversibility since a history cannot be reversed. The parameters describing the plastic deformations are effectively time dependent, and they increase continuously. Furthermore, the force density-stretch relations concerning elastic deformation must be replaced by increments of force density and stretch for plastic deformation.

\subsection{Incremental stretch and yield function}

For an increment of applied load, the corresponding increment of force density is $\Delta t_{(k)(j)}$, and the increment of stretch is $\Delta s_{(k)(j)}$. The incremental stretch, $\Delta s_{(k)(j)}$, can be decomposed as

$$
\Delta s_{(k)(j)}=\Delta s_{(k)(j)}^{e}+\Delta s_{(k)(j)}^{p}
$$

in which $\Delta s_{(k)(j)}^{e}$ and $\Delta s_{(k)(j)}^{p}$ represent the incremental elastic and plastic stretches between the material points.

With this decomposition, the incremental dilatation can be decomposed into elastic, $\Delta \theta_{(k)}^{e}$, and plastic, $\Delta \theta_{(k)}^{p}$, parts in the form

$$
\Delta \theta_{(k)}=\Delta \theta_{(k)}^{e}+\Delta \theta_{(k)}^{p},
$$

in which

$$
\Delta \theta_{(k)}^{e}=d \delta \sum_{j=1}^{N} \Delta s_{(k)(j)}^{e} \Lambda_{(k)(j)} V_{(j)}
$$

and 


$$
\Delta \theta_{(k)}^{p}=0
$$

under the assumption of zero dilatation due to plastic deformation.

If $: \ldots, \ldots$, represents the rate of plastic stretch at each instant of time, $t$, then the increment of plastic stretch in the time interval $\Delta t$ becomes $\Delta s_{(k)(j)}^{p}=i_{\ldots, \ldots,}$. The total plastic stretch after each successive yielding, $s_{(k)(j)}^{p}$, is the summation of the rate of plastic stretch as

$$
S_{(k)(j)}^{p}=S_{(k)(j)}^{p}(t=0)+\int_{0}^{t}: \ldots, \ldots, \ldots
$$

Under the assumption of yielding commencing when the distortional component of strain energy density, Eq. (13), reaches the strain energy due to the uniaxial tensile stress, $\sigma_{Y}$, the initial yield condition can be stated as

$$
\sigma_{Y}=\sqrt{6 \mu W_{(k)}^{\mu}},
$$

in which $\sigma_{Y}$ represents the initial yield stress of the material.

Substituting for the distortional part of the strain energy from (12), the yield condition can be rewritten as

$$
b \sum_{j=1}^{N} \delta s_{(k)(j)}^{2}\left|\mathbf{x}_{(j)}-\mathbf{x}_{(k)}\right| V_{(j)}-a_{\mu} \theta_{(k)}^{2}=\frac{\sigma_{Y}^{2}}{6 \mu}
$$

Thus, the yield function with strain hardening can be expressed as

$$
F_{(k)}=W_{(k)}^{\mu}-G\left(\bar{s}_{(k)}^{p}\right),
$$

where $G\left(\bar{s}_{(k)}^{p}\right)$ represents the final state of strain hardening, and its value may vary from point to point throughout the body. Its form depends on the strain hardening model. For a linear isotropic strain hardening model, it can be defined as

$$
G\left(\bar{s}_{(k)}^{p}\right)=\frac{\left(\sigma_{Y}+K \bar{s}_{(k)}^{p}\right)^{2}}{6 \mu},
$$

in which $\bar{s}_{(k)}^{p}$ and $K$ represent the equivalent plastic stretch and tangent modulus of the material, respectively. 


\subsection{Strain hardening and flow rule}

Provided that $F_{(k)}\left(t_{(k)(j)}\right)=0$, concerning an increment of force density, $\Delta t_{(k)(j)}$, the rate of change of the yield function and its value dictates loading, neutral, and unloading states. Thus, unloading occurs for

$$
\Delta F_{(k)}=\frac{1}{4} \sum_{j=1}^{N}\left(\frac{\partial F_{(k)}}{\partial t_{(k)(j)}} \Delta t_{(k)(j)}+\frac{\partial F_{(k)}}{\partial t_{(j)(k)}} \Delta t_{(j)(k)}\right)<0 .
$$

Otherwise, loading occurs if

$$
\Delta F_{(k)}=\frac{1}{4} \sum_{j=1}^{N}\left(\frac{\partial F_{(k)}}{\partial t_{(k)(j)}} \Delta t_{(k)(j)}+\frac{\partial F_{(k)}}{\partial t_{(j)(k)}} \Delta t_{(j)(k)}\right)>0,
$$

or neutral loading occurs if

$$
\Delta F_{(k)}=\frac{1}{4} \sum_{j=1}^{N}\left(\frac{\partial F_{(k)}}{\partial t_{(k)(j)}} \Delta t_{(k)(j)}+\frac{\partial F_{(k)}}{\partial t_{(j)(k)}} \Delta t_{(j)(k)}\right)=0 .
$$

For the state of unloading, there is no change in plastic deformation, i.e.,

$$
\frac{d s_{(k)(j)}^{p}}{d t}=: \ldots
$$

In the case of ideal plastic deformation without strain hardening, the yield function, $F_{(k)}$, is not affected by $s_{(k)(j)}^{p}$, thus it is always neutral during loading from a plastic state.

The strain energy density increment in the transfer from the plastic state of $t_{(k)(j)}$ and $s_{(k)(j)}$ to another plastic state of $t_{(k)(j)}+\Delta t_{(k)(j)}$ and $s_{(k)(j)}+\Delta s_{(k)(j)}$ can be expressed as

$$
\Delta W_{(k)}=\Delta W_{(k)}^{e}+\Delta W_{(k)}^{p},
$$

where

$$
\Delta W_{(k)}^{e}=\frac{1}{4} \sum_{j=1}^{\infty}\left(\Delta t_{(k)(j)} \Delta s_{(k)(j)}^{e}+\Delta t_{(j)(k)} \Delta s_{(j)(k)}^{e}\right) V_{(j)}
$$

and 


$$
\Delta W_{(k)}^{p}=\frac{1}{4} \sum_{j=1}^{\infty}\left(\Delta t_{(k)(j)} \Delta s_{(k)(j)}^{p}+\Delta t_{(j)(k)} \Delta s_{(j)(k)}^{p}\right) V_{(j)} .
$$

According to Drucker (1951), $\Delta W_{(k)}^{p}>0$ represents strain hardening and $\Delta W_{(k)}^{p}=0$ represents ideal plastic deformation without strain hardening. For ideal plastic deformation, Eqs. (21c) and (24b) can be rewritten in a slightly different form as

$$
\Delta F_{(k)}=\frac{1}{4} \sum_{j=1}^{N}\left(\frac{\partial F_{(k)}}{\partial t_{(k)(j)}} \Delta t_{(k)(j)}+\frac{\partial F_{(k)}}{\partial t_{(j)(k)}} \Delta t_{(j)(k)}\right)=0,
$$

or

$$
\Delta F_{(k)}=\frac{1}{4}\left(\left\{\begin{array}{c}
\frac{\partial F_{(k)}}{\partial t_{(k)(1)}} \\
\cdot \\
\cdot \\
\cdot \\
\frac{\partial F_{(k)}}{\partial t_{(k)(N)}}
\end{array}\right\} \cdot\left\{\begin{array}{c}
\Delta t_{(k)(1)} \\
\cdot \\
\cdot \\
\cdot \\
\Delta t_{(k)(N)}
\end{array}\right\}+\left\{\begin{array}{c}
\frac{\partial F_{(k)}}{\partial t_{(1)(k)}} \\
\cdot \\
\cdot \\
\cdot \\
\frac{\partial F_{(k)}}{\partial t_{(N)(k)}}
\end{array}\right\} \cdot\left\{\begin{array}{c}
\Delta t_{(1)(k)} \\
\cdot \\
\cdot \\
\cdot \\
\Delta t_{(N)(k)}
\end{array}\right\}=0,\right.
$$

and

$$
\Delta W_{(k)}^{p}=\frac{1}{4} \sum_{j=1}^{\infty}\left(\Delta t_{(k)(j)} \Delta s_{(k)(j)}^{p}+\Delta t_{(j)(k)} \Delta s_{(j)(k)}^{p}\right) V_{(j)}=0,
$$

or

$$
\Delta W_{(k)}^{p}=\frac{1}{4}\left(\left\{\begin{array}{c}
\Delta s_{(k)(1)}^{p} V_{(1)} \\
\cdot \\
\cdot \\
\cdot \\
\Delta s_{(k)(N)}^{p} V_{(N)}
\end{array}\right\} \cdot\left\{\begin{array}{c}
\Delta t_{(k)(1)} \\
\cdot \\
\cdot \\
\cdot \\
\Delta t_{(k)(N)}
\end{array}\right\}+\left\{\begin{array}{c}
\Delta s_{(1)(k)}^{p} V_{(1)} \\
\cdot \\
\cdot \\
\cdot \\
\Delta s_{(N)(k)}^{p} V_{(N)}
\end{array}\right\} \cdot\left\{\begin{array}{c}
\Delta t_{(1)(k)} \\
\cdot \\
\cdot \\
\cdot \\
\Delta t_{(N)(k)}
\end{array}\right\}\right)=0 .
$$

Note that the dot products of the vectors concerning the yield function and stretch with the vector of incremental tractions in Eq. (25b) and (25d) vanish. It is observed that these vectors concerning the yield function and stretch must be parallel to each other. Therefore, the following relation can be obtained: 


$$
\left\{\begin{array}{c}
\Delta s_{(k)(1)}^{p} V_{(1)} \\
\cdot \\
\cdot \\
\cdot \overrightarrow{\Delta s_{(k)(N)}^{p} V_{(N)}}
\end{array}\right\}=C_{(k)}\left\{\begin{array}{c}
\frac{\partial F_{(k)}}{\partial t_{(k)(1)}} \\
\cdot \\
\cdot \\
\cdot \\
\frac{\partial F_{(k)}}{\partial t_{(k)(N)}}
\end{array}\right\}
$$

and

$$
\left\{\begin{array}{c}
\Delta s_{(1)(\mathrm{k})}^{p} V_{(1)} \\
\cdot \\
\cdot \\
\cdot \\
\Delta s_{(N)(k)}^{p} V_{(N)}
\end{array}\right\}=C_{(k)}\left\{\begin{array}{c}
\frac{\partial F_{(k)}}{\partial t_{(1)(k)}} \\
\cdot \\
\cdot \\
\cdot \\
\frac{\partial F_{(k)}}{\partial t_{(N)(k)}}
\end{array}\right\} .
$$

For each interaction between material points $\mathbf{x}_{(k)}$ and $\mathbf{x}_{(j)}$, this relationship can be recast as

$$
\Delta s_{(k)(j)}^{p}=\frac{1}{V_{(j)}} C_{(k)} \frac{\partial F_{(k)}}{\partial t_{(k)(j)}},
$$

or

$$
\Delta s_{(k)(j)}^{p}=\frac{1}{V_{(j)}} C_{(k)} \frac{\partial F_{(k)}}{\partial t_{(k)(j)}^{\mu}} .
$$

Substituting for the yield function, $F_{(k)}$, and performing the necessary algebraic manipulations result in the expression for the incremental plastic stretch as

$$
\Delta s_{(k)(j)}^{p}=C_{(k)}\left(\frac{1}{2 \delta b}\left|\mathbf{x}_{(j)}-\mathbf{x}_{(k)}\right| t_{(k)(j)}+\frac{d}{b} a_{\mu}\left(1-\frac{a_{\kappa}}{a_{\mu}}\right) \Lambda_{(k)(j)} \theta_{(k)}\right)
$$

where $C_{(k)}$ is a positive proportionality constant. This expression provides the rule for incremental plastic stretch, and serves as the normality condition. 


\subsection{Equivalent stress-equivalent plastic stretch}

The relationship given by Eq. (17) describes the yield surface for the loading path of uniaxial loading only. If the loading path is different than that of uniaxial loading, the yield surface is constructed based on the concept of equivalent stress and equivalent plastic stretch. The equivalent stress can be defined as

$$
\bar{\sigma}_{e(k)}=\sqrt{6 \mu W_{(k)}^{\mu}\left(s_{(k)(j)}\right)},
$$

or

$$
\bar{\sigma}_{e(k)}=\sqrt{6 \mu}\left[b \delta \sum_{j=1}^{N} s_{(k)(j)}^{2}\left|\mathbf{x}_{(j)}-\mathbf{x}_{(k)}\right| V_{(j)}-a_{\mu} \theta_{(k)}^{2}\right]^{1 / 2} .
$$

An equivalent plastic stretch increment, $\Delta \bar{s}_{(k)}^{p}$, can be defined as

$$
\Delta \bar{s}_{(k)}^{p}=A_{0} \sqrt{W_{(k)}^{\mu}\left(\Delta s_{(k)(j)}^{p}\right)}
$$

in which $W_{(k)}^{\mu}\left(\Delta s_{(k)(j)}^{p}\right)$ represents the distortional part of the strain energy density due to the incremental plastic stretch, $\Delta s_{(k)(j)}^{p}$. As described in Appendix B, the parameter $A_{0}$ is determined in such a way that $\Delta \bar{s}_{(k)}^{p}$ recovers the incremental plastic stretch due to uniaxial tension. According to the dimension of the analysis, it can be determined as

$$
\begin{aligned}
& A_{0}=\frac{1}{\sqrt{A b \delta^{3}}} \text { for }(1-\mathrm{D}), \\
& A_{0}=\frac{\sqrt{3}}{\sqrt{\pi b h \delta^{4}}} \text { for }(2-\mathrm{D}), \\
& A_{0}=\sqrt{\frac{5}{\pi b \delta^{5}}} \text { for }(3-\mathrm{D}) .
\end{aligned}
$$

After substituting for $W_{(k)}^{\mu}\left(\Delta s_{(k)(j)}^{p}\right)$ from Eq.(12) and invoking $\Delta \theta_{(k)}^{p}=0$, the equivalent plastic stretch increment can be expressed as

$$
\Delta \bar{s}_{(k)}^{p}=A_{0} \sqrt{b \delta \sum_{j=1}^{N}\left(\Delta s_{(k)(j)}^{p}\right)^{2}\left|\mathbf{x}_{(j)}-\mathbf{x}_{(k)}\right| V_{(j)}} .
$$




\section{Imposition of boundary conditions}

The constraint conditions are, in general, not necessary for the solution of an integrodifferential equation because the PD equations of motion do not contain any spatial derivatives. Unlike the local theory, the boundary conditions are imposed through a nonzero volume of fictitious boundary layers. Based on numerical experiments, Macek and Silling (2007) suggested that the extent of the fictitious boundary layer be equal to the horizon, $\delta$, in order to ensure that the imposed prescribed constraints are accurately reflected in the real domain.

The displacement boundary conditions can be imposed by assigning constraints to the material points in the fictitious layer in such a way that the condition is satisfied explicitly on the boundary surface. Therefore, the value of the displacement in the fictitious layer is approximated based on the linear extrapolation of the values in the real domain and the specified value of the boundary condition. Similarly, the traction boundary conditions can be enforced by approximating the value of the traction in the fictitious region, so that the variation of the traction in both the real domain and the fictitious layer recovers the applied traction on the boundary surface. However, in the case of zero traction, it is not necessary to employ a fictitious layer. Hence, it can be satisfied in a natural way without enforcing any specific constraints.

\subsection{Displacement constraints}

In the case of a two-dimensional analysis, the displacement constraints concern the imposition of a prescribed value of the displacement components, $U^{*}$ and $V^{*}$, on the boundary. This type of boundary condition can be achieved through a fictitious region, $R_{f}$. Therefore, a fictitious boundary layer with depth $\delta$ is introduced along the boundary of the actual material region, $R$, as shown in Fig. 3.

The prescribed boundary value of the displacements $U^{*}$ and $V^{*}$ in the $x$ - and $y$-directions

can be imposed through a layer of the fictitious region, $R_{f}$, along the boundary of the material surface, $S$, as

$$
\begin{aligned}
& u_{f}\left(x_{f}, y_{f}, t+\Delta t\right)=2 U^{*}\left(x^{*}, y^{*}, t+\Delta t\right)-u(x, y, t), \\
& v_{f}\left(x_{f}, y_{f}, t+\Delta t\right)=2 V^{*}\left(x^{*}, y^{*}, t+\Delta t\right)-v(x, y, t),
\end{aligned}
$$

which represent the clamped boundary conditions for the prescribed values of $U^{*}=V^{*}=0$ (Fig. $3 b)$.

If only the displacement constraint $U^{*}$ is applied (i.e., no displacement constraint in the other direction), the boundary conditions can be imposed as

$$
\begin{aligned}
& u_{f}\left(x_{f}, y_{f}, t+\Delta t\right)=2 U^{*}\left(x^{*}, y^{*}, t+\Delta t\right)-u(x, y, t), \\
& v_{f}\left(x_{f}, y_{f}, t+\Delta t\right)=v(x, y, t),
\end{aligned}
$$

which represents the roller support boundary conditions for the prescribed value, $U^{*}=0$, (Fig. 4). 


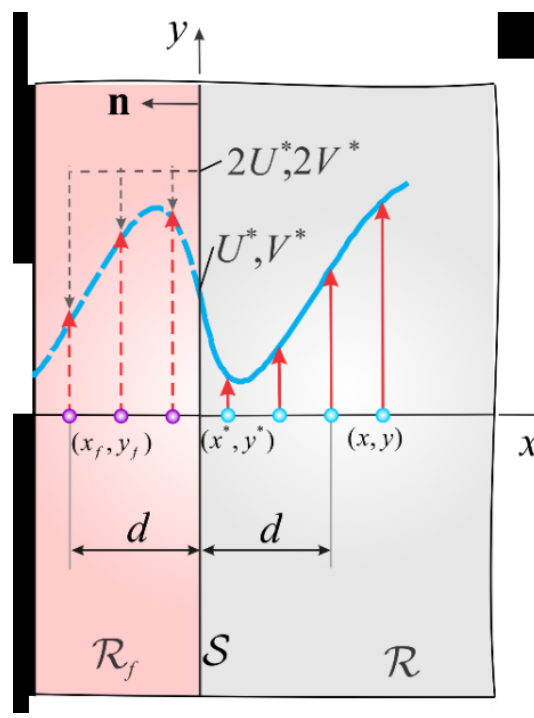

(a)

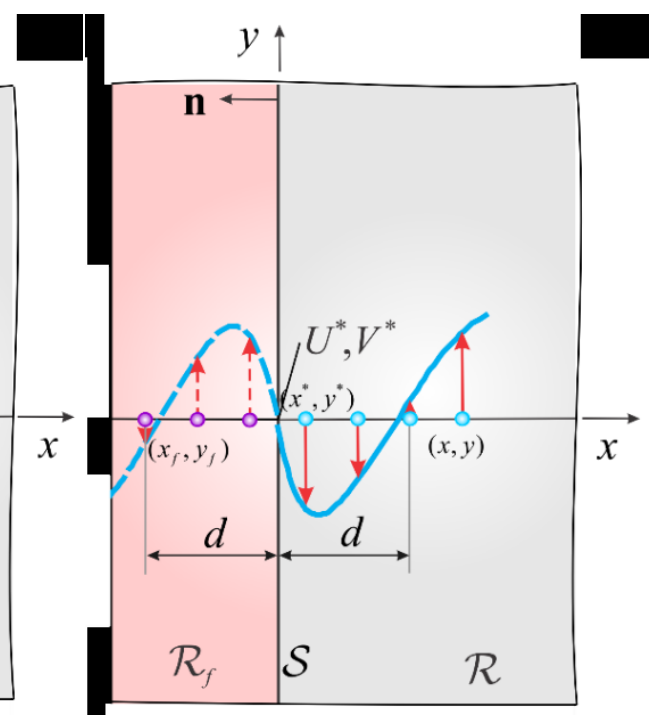

(b)

Fig. 3. Imposing boundary condition on displacement components: (a) constant displacement components $u\left(x^{*}, y^{*}, t\right)=U^{*}$ and $v\left(x^{*}, y^{*}, t\right)=V^{*}$; (b) zero displacement components $u\left(x^{*}, y^{*}, t\right)=U^{*}=0$ and $v\left(x^{*}, y^{*}, t\right)=V^{*}=0$.

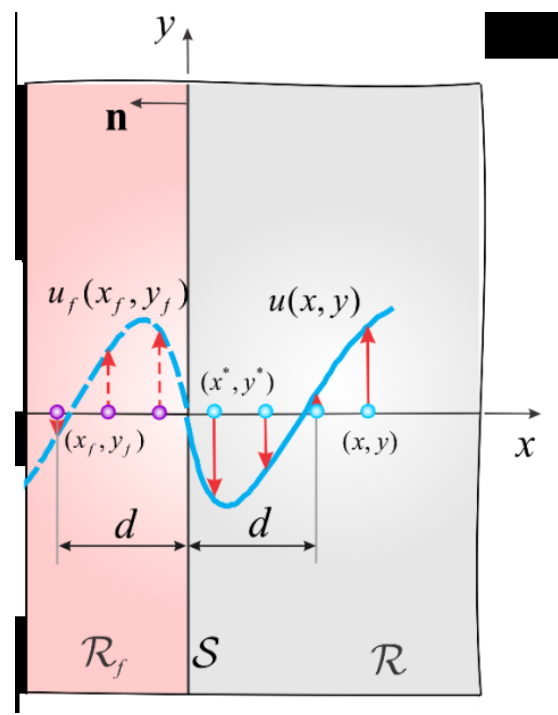

(a)

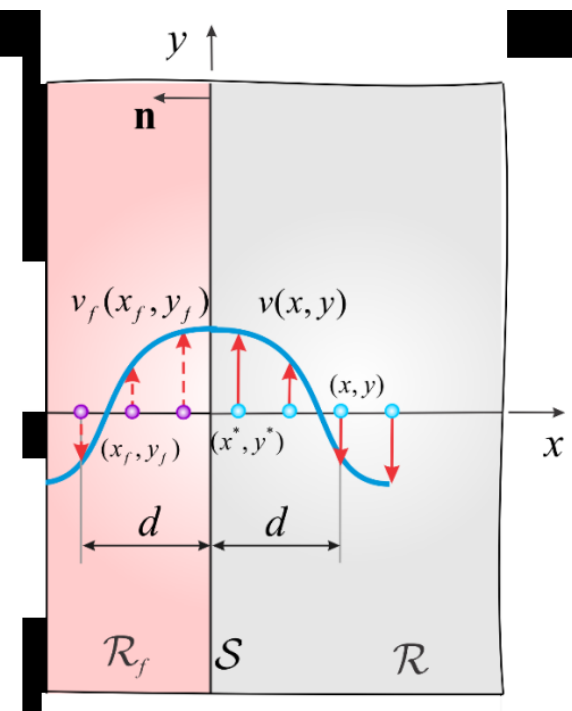

(b)

Fig. 4. Imposing boundary condition on displacement components: (a) fixed in the normal direction $U^{*}=0$; (b) free to move in the tangent direction .

\subsection{Traction boundary conditions}

Similar to the essential boundary conditions, the traction boundary conditions can be imposed through a fictitious region, $R_{f}$. On the boundary with a unit normal $\mathbf{n}=\mathbf{e}_{x}$, the applied stresses, 
$\sigma_{0}$ and $\tau_{0}$, normal and tangent to the boundary can be enforced by assigning the following displacement values in the fictitious region:

$$
\begin{aligned}
& u_{f}\left(x_{f}, y_{f}, t+\Delta t\right)=\left[\frac{\sigma_{o}\left(1-v^{2}\right)}{E}-v \frac{v\left(x, y^{+}, t\right)-v\left(x, y^{-}, t\right)}{y^{+}-y^{-}}\right]\left(x_{f}-x\right)+u(x, y, t), \\
& v_{f}\left(x_{f}, y_{f}, t+\Delta t\right)=\left[\frac{2(1+v) \tau_{o}}{E}-\frac{u\left(x, y^{+}, t\right)-u\left(x, y^{-}, t\right)}{y^{+}-y^{-}}\right]\left(x_{f}-x\right)+v(x, y, t),
\end{aligned}
$$

in which $(x, y)$ are the coordinates of a material point in the real boundary layer, $R$. As shown in Fig. 5a, the locations of material points above and below this point are denoted by $\left(x, y^{+}\right)$and $\left(x, y^{-}\right)$, respectively.

On the boundary with a unit normal $\mathbf{n}=\mathbf{e}_{y}$, the applied stresses, $\sigma_{0}$ and $\tau_{0}$, normal and tangent to the boundary can be enforced in the fictitious region as

$$
\begin{aligned}
& u_{f}\left(x_{f}, y_{f}, t+\Delta t\right)=\left[\frac{2(1+v) \tau_{o}}{E}-\frac{v\left(x^{+}, y, t\right)-v\left(x^{-}, y, t\right)}{x^{+}-x^{-}}\right]\left(y_{f}-y\right)+u(x, y, t), \\
& v_{f}\left(x_{f}, y_{f}, t+\Delta t\right)=\left[\frac{\sigma_{o}\left(1-v^{2}\right)}{E}-v \frac{u\left(x^{+}, y, t\right)-u\left(x^{-}, y, t\right)}{x^{+}-x^{-}}\right]\left(y_{f}-y\right)+v(x, y, t) .
\end{aligned}
$$

As shown in Fig. 5b, the locations of the material points on the left and on the right of this point are denoted by $\left(x^{-}, y\right)$ and $\left(x^{+}, y\right)$, respectively. Note this procedure is only applicable if the boundary region is elastic. If both normal and tangential stress components are zero along the boundary, a fictitious region is not necessary as they are satisfied in a natural way. The robustness of this approach is demonstrated in Appendix C. Alternative to this approach is the representation of the applied traction in the form of a body force, and invoking it into the equation of motion as described by Madenci and Oterkus (2014).

\section{Failure prediction}

A failure process is included in the analysis by monitoring the interaction between two material points, $\mathbf{x}$ and $\mathbf{x}^{\prime}$. Their interaction can be terminated based on a particular criteria; thus, leading to the removal of their peridynamic force from the equations of motion by introducing the function $\mu\left(\mathbf{x}^{\prime}-\mathbf{x}, t\right)$ as 


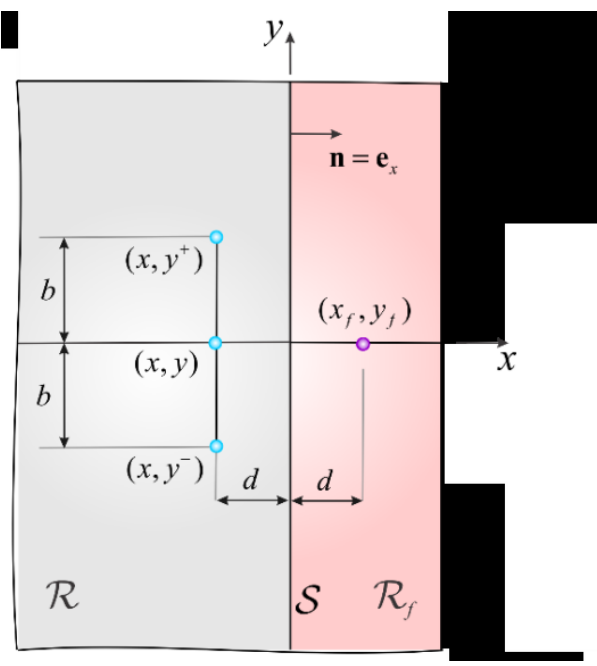

(a)

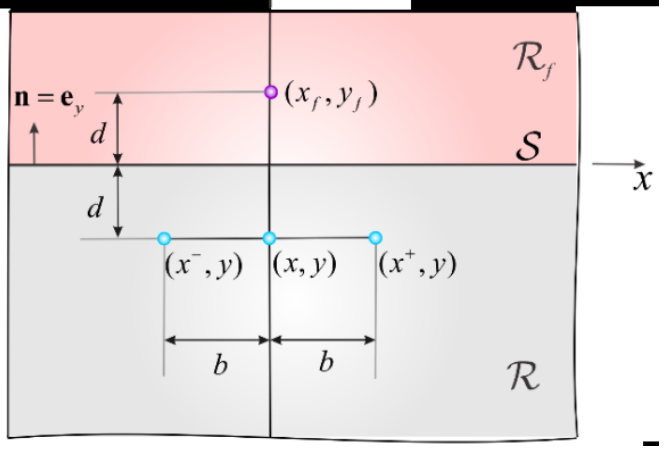

(b)

Fig. 5. Fictitious region along the boundary with a unit normal $\mathbf{n}=\mathbf{e}_{x}$ (a) and $\mathbf{n}=\mathbf{e}_{y}$ (b).

$$
\rho(\mathbf{x})^{\cdot} \cdot \quad, \quad \int_{H} \mu\left(\mathbf{x}^{\prime}-\mathbf{x}, t\right)\left(\mathbf{t}\left(\mathbf{u}^{\prime}-\mathbf{u}, \mathbf{x}^{\prime}-\mathbf{x}, t\right)-\mathbf{t}^{\prime}\left(\mathbf{u}^{\prime}-\mathbf{u}, \mathbf{x}^{\prime}-\mathbf{x}, t\right)\right) d H+\mathbf{b}(\mathbf{x}, t),
$$

where

$$
\mu\left(\mathbf{x}^{\prime}-\mathbf{x}, t\right)=\left\{\begin{array}{l}
1 \text { if interaction exists } \\
0 \text { if no interaction }
\end{array}\right.
$$

For the case of elastic deformation, Silling (2000) introduced a criteria based on the critical stretch value. According to his criteria, the interaction between two material points, $\mathbf{x}_{(k)}$ and $\mathbf{x}_{(j)}$ , is terminated once the stretch between them, $s_{(k)(j)}$, reaches its critical value, $s_{c}$. Thus, the peridynamic force density between these material points vanishes and the critical stretch can be related to critical energy release rate, $G_{c}$, of the material (Silling and Askari, 2005; Madenci and Oterkus, 2014) as

$$
s_{c}= \begin{cases}\sqrt{\frac{G_{c}}{\left.3 \mu+\left(\frac{3}{4}\right)^{4}\left(\kappa-\frac{5 \mu}{3}\right)\right) \delta}} & \text { three dimensions, } \\ \sqrt{\frac{G_{c}}{\left(\frac{6}{\pi} \mu+\frac{16}{9 \pi^{2}}(\kappa-2 \mu)\right) \delta}} & \text { two dimensions. }\end{cases}
$$


For the case of plastic deformation, the force density between two material points, $\mathbf{x}_{(k)}$ and $\mathbf{x}_{(j)}$, exhibits a nonlinear behavior, as shown in Fig. 6. The area under this curve represents the elastic-plastic micropotential, $w_{(k)(j)}$, reflecting the degree of plastic deformation. It can be determined as

$$
w_{(k)(j)}=\int_{0}^{s_{(k)(j)}} t_{(k)(j)}\left|\mathbf{x}_{j}-\mathbf{x}_{k}\right| d s_{(k)(j)} .
$$

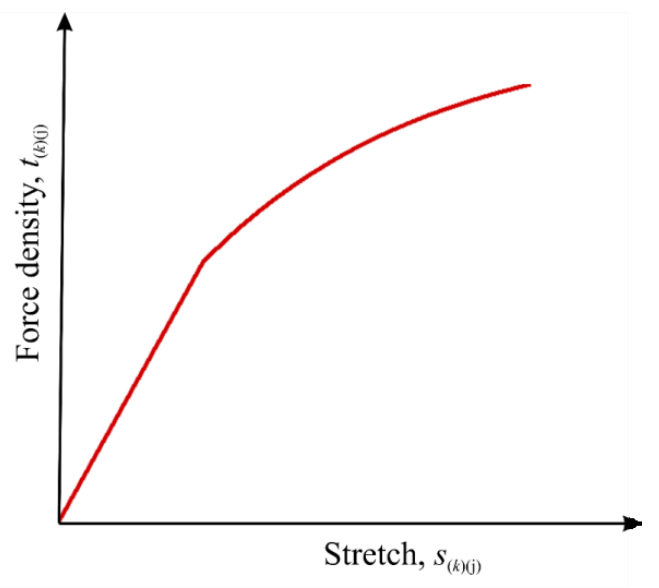

Fig. 6. Constitutive relation between material points .

In order to create a new crack surface, $A$, all of the micropotentials between the material points $\mathbf{x}_{\left(k^{+}\right)}$and $\mathbf{x}_{\left(j^{-}\right)}$whose line of action crosses this new surface must be terminated (Fig. 7). The material points $\mathbf{x}_{\left(k^{+}\right)}$and $\mathbf{x}_{\left(j^{-}\right)}$are located above and below the new crack surface, respectively. The number of material points within the family of $\mathbf{x}_{\left(k^{+}\right)}$below the crack surface and intersecting with the crack is denoted by $K^{-}$. Similarly, $J^{+}$represents the number of material points above the crack surface within the family of $\mathbf{x}_{\left(j^{-}\right)}$and intersecting with the crack. 


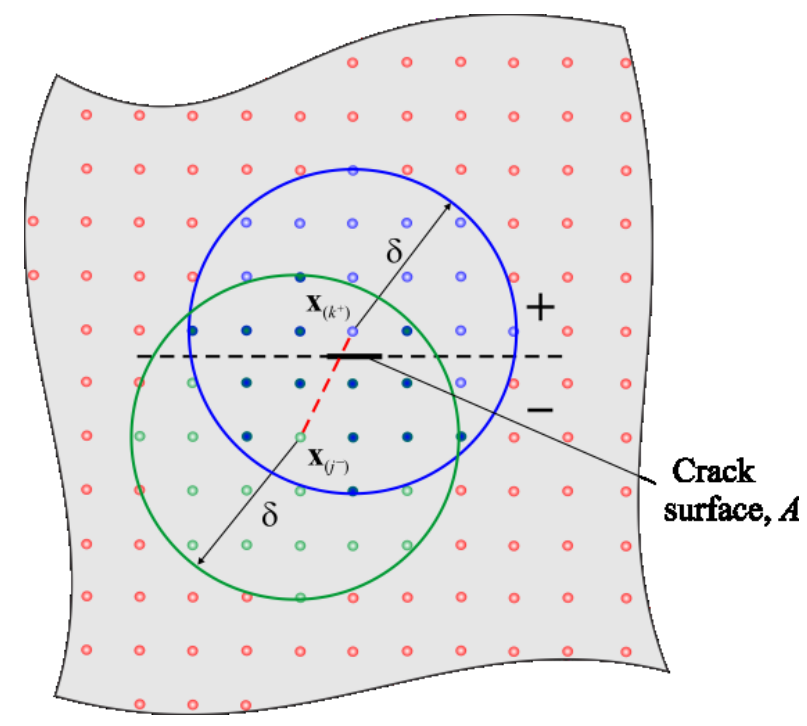

Fig. 7. Interaction across a crack surface between material points $\mathbf{x}_{\left(k^{+}\right)}$and $\mathbf{x}_{\left(j^{-}\right)}$.

The strain energy required to remove the interaction between two material points, $\mathbf{x}_{\left(k^{+}\right)}$and $\mathbf{x}_{\left(j^{-}\right)}$, can be expressed as

$$
W_{\left(k^{+}\right)\left(j^{-}\right)}=\frac{1}{2} \frac{w_{\left(k^{+}\right)\left(j^{-}\right)}+w_{\left(j^{-}\right)\left(k^{+}\right)}}{2} V_{\left(k^{+}\right)} V_{\left(j^{-}\right)}
$$

Furthermore, the total strain energy required to remove all of the interactions across the crack surface $A$ can be obtained as

$$
W=\frac{1}{2} \sum_{k=1}^{K^{+}} \frac{1}{2} \sum_{j=1}^{J^{-}} w_{\left(k^{+}\right)\left(j^{-}\right)} V_{\left(k^{+}\right)} V_{\left(j^{-}\right)}+\frac{1}{2} \sum_{k=1}^{K^{+}} \frac{1}{2} \sum_{j=1}^{J^{-}} w_{\left(j^{-}\right)\left(k^{+}\right)} V_{\left(j^{-}\right)} V_{\left(k^{+}\right)},
$$

for which the line of interaction defined by $\left|\mathbf{x}_{\left(k^{+}\right)}-\mathbf{x}_{\left(j^{-}\right)}\right|$and the crack surface intersect, and $K^{+}$ indicates the number of material points above and $J^{-}$the number below the crack surface within the families of $\mathbf{x}_{\left(k^{+}\right)}$and $\mathbf{x}_{\left(j^{-}\right)}$, respectively. If this line of interaction and crack surface intersect at the crack tip, only half of the critical micropotential is considered in the summation. This expression can be simplified as

$$
W=\frac{1}{2} \sum_{k=1}^{K^{+}} \sum_{j=1}^{J^{-}} \frac{1}{2}\left(w_{\left(k^{+}\right)\left(j^{-}\right)}+w_{\left(j^{-}\right)\left(k^{+}\right)}\right) V_{\left(k^{+}\right)} V_{\left(j^{-}\right)} .
$$

Substituting for micropotentials given by Eq. (40) in Eq. (43) results in the strain energy required to eliminate all of the interactions across the unit crack surface, $A=h \Delta x$, with $\Delta x$ representing the spacing between the material points and $h$ the thickness. 
The total work, $W$, required to eliminate all interactions across this new surface can be related to the value J-integral, $J$ (nonlinear energy release rate) (Rice, 1968; Begley and Landes, 1972) as

$$
J=\frac{1}{2} \sum_{k=1}^{K^{+}} \sum_{j=1}^{J^{-}} \frac{1}{2}\left(J_{\left(k^{+}\right)\left(j^{-}\right)}+J_{\left(j^{-}\right)\left(k^{+}\right)}\right),
$$

where

$$
\begin{aligned}
& J_{\left(k^{+}\right)\left(j^{-}\right)}=\frac{1}{(\Delta x) h} w_{\left(k^{+}\right)\left(j^{-}\right)} V_{\left(k^{+}\right)} V_{\left(j^{-}\right)}, \\
& J_{\left(j^{-}\right)\left(k^{+}\right)}=\frac{1}{(\Delta x) h} w_{\left(j^{-}\right)\left(k^{+}\right)} V_{\left(k^{+}\right)} V_{\left(j^{-}\right)}
\end{aligned}
$$

For a horizon size of $\delta=3 \Delta x$, the number of material points above and below the crack surfaces is $K^{+}=J^{-}=9$. The total number of interactions crossing the crack surface is $2 \times 22$, and $2 \times 10$ of these interactions intersect the crack at the tip, as shown in Fig. 8. The number of interactions creating a unit crack surface can be calculated as $N_{c}=(2 \times 22)-(2 \times 10) / 2=34$. The measured critical value, $J_{c}$, of the material defines the amount of energy required to remove all interactions, leading to

$$
J_{c}=\frac{1}{2} \sum_{k=1}^{K^{+}} \sum_{j=1}^{J^{-}} \frac{1}{2}\left(J_{\left(k^{+}\right)\left(j^{-}\right)}^{c}+J_{\left(j^{-}\right)\left(k^{+}\right)}^{c}\right) .
$$

Peridynamics enables the simulation of damage propagation by progressively removing the interactions between materials points. In order to reflect this feature, the critical energy release rate, $J_{c}$, of a material point is distributed equally to each interaction at that material point (Hu et al., 2014). Note that the explicit determination of the critical stretch, $s_{c}$, is not required in the numerical implementation of the peridynamic analysis. Under the assumption that $J_{\left(k^{+}\right)\left(j^{-}\right)}^{c} \approx J_{\left(j^{-}\right)\left(k^{+}\right)}^{c}$, the failure criteria for each interaction can be expressed as

$$
\frac{J_{(k)(j)}}{J_{(k)(j)}^{c}} \geq 1
$$

with

$$
J_{\left(k^{+}\right)\left(j^{-}\right)}^{c}=\frac{J_{c}}{N_{c}} .
$$

A similar energy based failure criteria was used by Foster et al. (2011) as part of the non-ordinary state-based peridynamic analysis of a crack growth in a material that exhibits viscoplastic behavior. 
As derived by Silling and Lehoucq (2010) and Hu et al. (2012), the PD J integral value around the crack tip can also be calculated as

$$
J_{p e r i}(\mathbf{x})=\int_{\partial R} W(x ; a) n_{1} d S-\frac{1}{2} \int_{R(a)}\left\{\int_{\Omega(a) \backslash \mathrm{R}(\mathrm{a})}\left(\mathbf{t}-\mathbf{t}^{\prime}\right) \cdot\left(\frac{\partial \mathbf{u}}{\partial x}+\frac{\partial \mathbf{u}^{\prime}}{\partial x}\right) h d A^{\prime}\right\} d A,
$$

where $W$ is the strain energy density, $n_{1}$ is the outward unit normal, and $d S$ is the length increment around the contour, $\Gamma$. The integration path around the crack tip is defined by $\partial R$. The region inside the integration path is $R(a)$, and the region outside the integration path is $\Omega(a) \backslash R(a)$, as shown in Fig. 9.
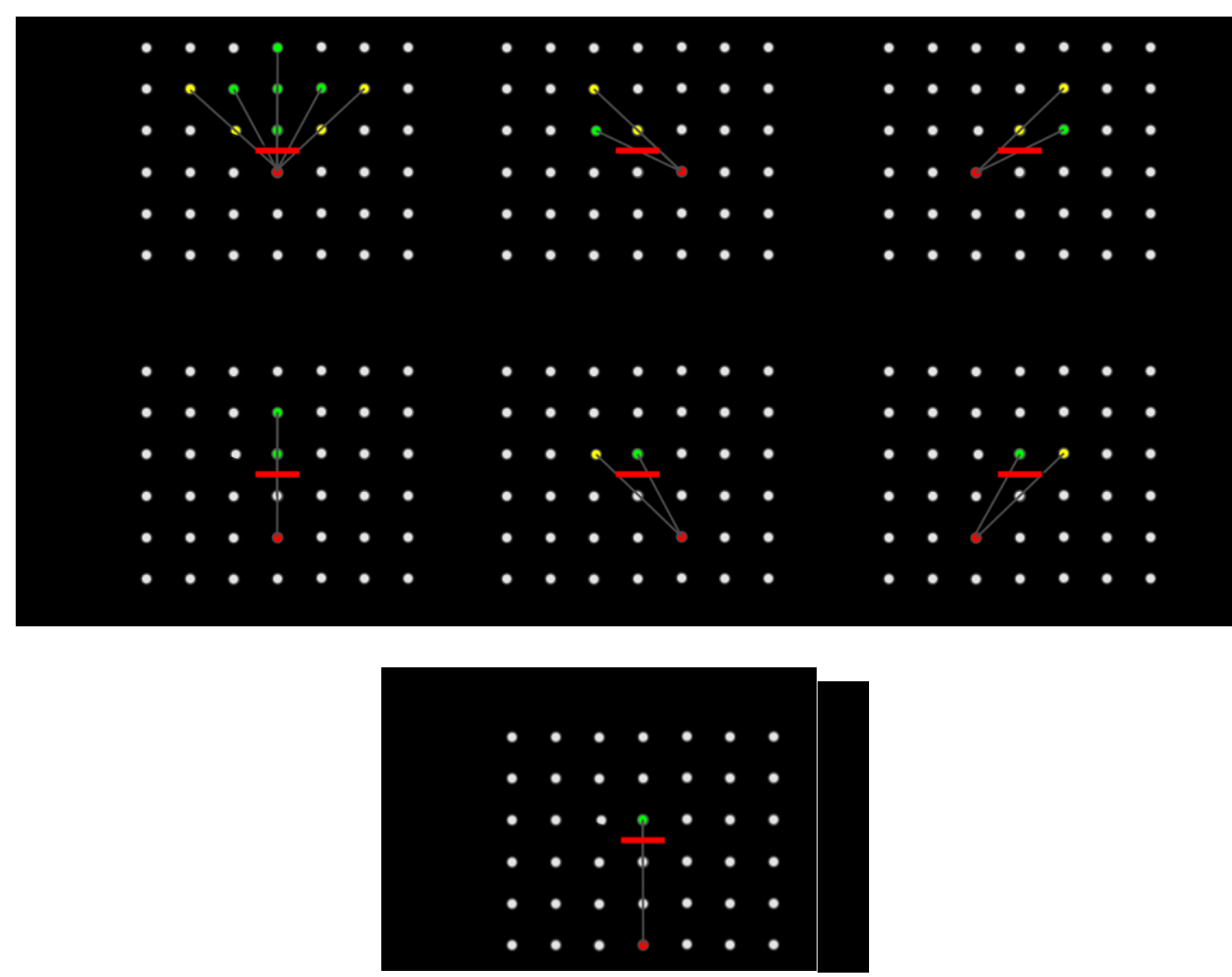

Fig. 8. Interactions of material points above the crack surface interacting with the material points below the crack surface. 


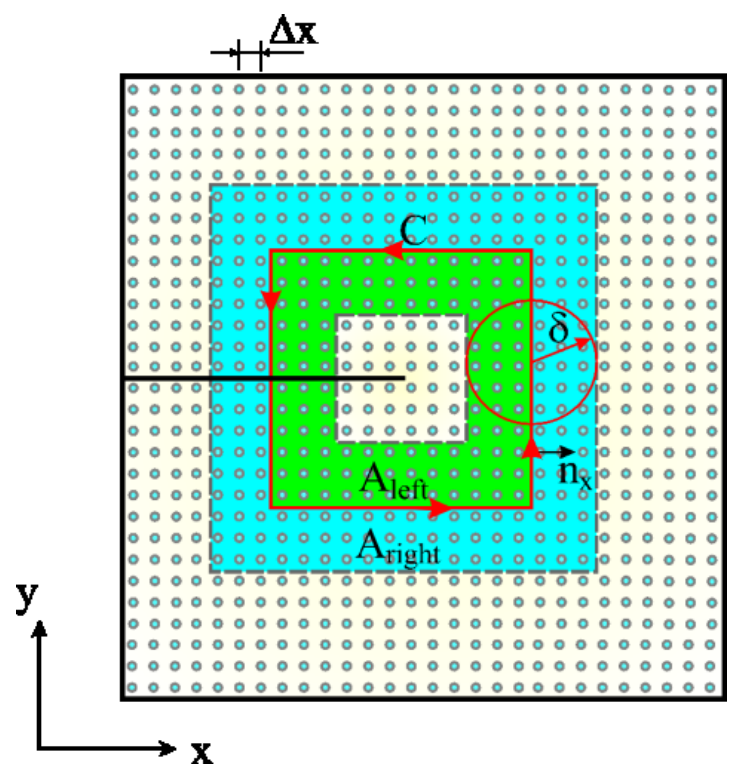

Fig. 9. Areas for peridynamic J-integral.

In discretized form, the J-integral can be expressed as

$$
\begin{aligned}
& J_{\text {peri }}=\sum_{i=1}^{n_{\text {contour }}} W_{(i)} n_{(i) x} \Delta x-\frac{1}{2} \sum_{j=1}^{n_{\text {left }}} \sum_{k=1}^{n_{\text {right }}}\left(\mathbf{t}_{(k)(j)}-\mathbf{t}_{(j)(k)}\right) \cdot\left(\frac{\partial \mathbf{u}_{(j)}}{\partial x}+\frac{\partial \mathbf{u}_{(k)}}{\partial x}\right) h A_{(k)} A_{(j)}, \\
& \frac{\partial \mathbf{u}}{\partial x}=\left\{\begin{array}{l}
\frac{\partial u_{1}\left(x_{1}, x_{2}\right)}{\partial x_{1}} \\
\frac{\partial u_{2}\left(x_{1}, x_{2}\right)}{\partial x_{1}}
\end{array}\right\}=\left\{\begin{array}{l}
\frac{u_{1}\left(x_{1}+\Delta x, x_{2}\right)-u_{1}\left(x_{1}-\Delta x, x_{2}\right)}{2 \Delta x} \\
\frac{u_{2}\left(x_{1}+\Delta x, x_{2}\right)-u_{2}\left(x_{1}-\Delta x, x_{2}\right)}{2 \Delta x}
\end{array}\right\} .
\end{aligned}
$$

Local damage, which defines the ratio of eliminated interactions to the total number of interactions associated with a material point within its horizon, can be calculated as (Silling and Askari, 2005)

$$
\varphi\left(\mathbf{x}^{\prime}-\mathbf{x}, t\right)=1-\frac{\int_{H} \mu\left(\mathbf{x}^{\prime}-\mathbf{x}, t\right) d V^{\prime}}{\int_{H} d V^{\prime}} .
$$

Local damage has a value between 0 and 1 . No damage at that material point is denoted by $\varphi=0$ , while $\varphi=1$ denotes that all the interactions are eliminated with the related material point. Moreover, $\varphi=0.5$ denotes that half of the interactions are eliminated with the related material point. 


\section{Numerical analysis}

The solution to the PD equation of motion requires spatial integration, which is performed by using a Gaussian integration (meshless) scheme. The domain is divided into a uniform grid, with material (integration or collocation) points associated with specific volumes. The time integration is achieved through an explicit scheme, in conjunction with the adaptive dynamic relaxation method described by Kilic and Madenci (2010). The solution to the equation of motion while satisfying the flow rule requires an iterative technique. Therefore, the external load is applied in an incremental manner and the following steps are performed in order to achieve convergence.

(1) At the $[\mathrm{n}+1]$ loading step, the stretch value is decomposed as

$$
S_{(k)(j)}^{[n+1]}=S_{(k)(j)}^{e[n+1]}+S_{(k)(j)}^{p[n+1]},
$$

or

$$
S_{(k)(j)}^{[n+1]}=S_{(k)(j)}^{[n]}+\left(\Delta s_{(k)(j)}^{[n+1]}\right),
$$

where

$$
\Delta s_{(k)(j)}^{[n+1]}=\left(\Delta s_{(k)(j)}^{e[n+1]}+\Delta s_{(k)(j)}^{p[n+1]}\right)
$$

in which $s_{(k)(j)}^{[n]}$ and $s_{(k)(j)}^{[n+1]}$ are known; however, $\Delta s_{(k)(j)}^{e[n+1]}$ and $\Delta s_{(k)(j)}^{p[n+1]}$ are unknown.

(2) Since $s_{(k)(j)}^{\mathrm{e}[n+1]}=s_{(k)(j)}^{[n+1]}-\left(s_{(k)(j)}^{\mathrm{p}[n]}+\Delta s_{(k)(j)}^{\mathrm{p}[n+1]}\right)$, an initial guess is assumed as elastic with zero plastic stretch increment, leading to

$$
s_{(k)(j)(t r i a l)}^{e[n+1]}=s_{(k)(j)}^{[n+1]}-\left(s_{(k)(j)}^{\mathrm{p}[n]}\right) \text { with } \Delta s_{(k)(j)}^{p[n+1]}=0
$$

(3) The corresponding force density for each interaction is calculated as

$$
t_{(k)(j)(\text { trial })}^{[n+1]}=\frac{2 \delta d}{\left|\mathbf{x}_{(j)}-\mathbf{x}_{(k)}\right|}\left(a_{\kappa}-a_{\mu}\right) \Lambda_{(k)(j)} \theta_{(k)}\left(s_{(k)(j)(t r i a l)}^{e[n+1]}\right)+(2 \delta b) s_{(k)(j)(\text { trial })}^{e[n+1]} .
$$

(4) The corresponding yield function from Eq. (19) and effective stress from Eq. (29) for each material point are calculated as

$$
F_{(k)(\text { trial })}^{[n+1]}=b \sum_{j=1}^{N} \frac{\delta}{\left|\mathbf{x}_{(j)}-\mathbf{x}_{(k)}\right|}\left(s_{(k)(j)(\text { trial })}^{e[n+1]}\right)^{2}\left|\mathbf{x}_{(j)}-\mathbf{x}_{(k)}\right|^{2} V_{(j)}-a_{\mu}\left(\theta_{(k)(t r i a)}^{e[n+1]}\right)^{2}-G\left(\bar{s}_{(k)}^{[[n]}\right) .
$$

and 


$$
\sigma_{\mathrm{eff}(k)(\text { trial })}^{[n+1]}=\sqrt{6 \mu\left(b \sum_{j=1}^{N} \frac{\delta}{\left|\mathbf{x}_{(j)}-\mathbf{x}_{(k)}\right|}\left(s_{(k)(j)(\text { trial })}^{e n+1]}\right)^{2}\left|\mathbf{x}_{(j)}-\mathbf{x}_{(k)}\right|^{2} V_{(j)}-a_{\mu}\left(\theta_{(k)(\text { trial })}^{e[n+1}\right)^{2}\right)} .
$$

(4a) If $F_{(k)(\text { trial })}^{[n+1]} \leq 0$, the trial elastic stretch is correct; thus, $s_{(k)(j)}^{e[n+1]}=s_{(k)(j)(t r i a l)}^{e[n+1]}$.

(4b) If $F_{(k)(\text { trial })}^{[n+1]}>0$, the trial elastic stretch is not correct; thus, $\Delta s_{(k)(j)}^{p[n+1]} \neq 0$ whose value is yet to be determined.

Note that the magnitude of plastic stretch increment is dictated by $C_{(k)}$ as given by Eq. (28). Therefore, the elastic stretch increment is corrected as

$$
s_{(k)(j)}^{\mathrm{e}[n+1]}=s_{(k)(j)}^{[n+1]}-\left(s_{(k)(j)}^{\mathrm{p}[n]}+\Delta s_{(k)(j)}^{\mathrm{p}[n+1]}\right) .
$$

or

$$
s_{(k)(j)}^{\mathrm{e}[n+1]}=s_{(k)(j)(t r i a l)}^{e[n+1]}-\Delta s_{(k)(j)}^{\mathrm{p}[n+1]} .
$$

In accordance with Eq. (28), the yield function can be recast as

$$
F_{(k)}^{[n+1]}=b \sum_{j=1}^{N} \frac{\delta}{\left|\mathbf{x}_{(j)}-\mathbf{x}_{(k)}\right|}\left(s_{(k)(j)}^{\mathrm{e}[n+1]}\right)^{2}\left|\mathbf{x}_{(j)}-\mathbf{x}_{(k)}\right|^{2} V_{(j)}-a_{\mu}\left(\theta_{(k)}^{e[n+1]}\right)^{2}-G\left(\bar{S}_{(k)}^{p[n+1]}\right) .
$$

or

$$
\begin{aligned}
F_{(k)}^{[n+1]}= & b \sum_{j=1}^{N} \frac{\delta}{\left|\mathbf{x}_{(j)}-\mathbf{x}_{(k)}\right|}\left(s_{(k)(j)(t r i a l)}^{e[n+1]}-\Delta s_{(k)(j)}^{\mathrm{p}[n+1]}\right)^{2}\left|\mathbf{x}_{(j)}-\mathbf{x}_{(k)}\right|^{2} V_{(j)} \\
& -a_{\mu}\left(d \sum_{j=1}^{N} \delta\left(s_{(k)(j)(t r i a l)}^{e[n+1]}-\Delta s_{(k)(j)}^{\mathrm{p}[n+1]}\right) \Lambda_{(j)(k)} V_{(j)}\right)^{2}-G\left(\bar{s}_{(k)}^{p[n]}+\Delta \bar{s}_{(k)}^{p[n+1]}\right)
\end{aligned}
$$

in which $\Delta S_{(k)(j)}^{p[n+1]}$ and $\Delta \bar{s}_{(k)}^{p[n+1]}$ can be expressed as

$$
\Delta s_{(k)(j)}^{p[n+1]}=C_{(k)} B_{(k)(j)} .
$$

and

$$
\Delta \bar{S}_{(k)}^{p[\mathrm{n}+1]}=C_{(k)} A_{0} D_{(k)} .
$$

with 


$$
B_{(k)(j)}=\frac{1}{2 \delta b}\left(t_{(k)(j)(t r i a l)}^{[n+1]}\right)\left|\mathbf{x}_{(j)}-\mathbf{x}_{(k)}\right|+\frac{d}{b} a_{\mu}\left(1-\frac{a_{\kappa}}{a_{\mu}}\right) \theta_{(k)}\left(s_{(k)(j)(t r i a l)}^{e[n+1]}\right) \Lambda_{(k)(j)} .
$$

and

$$
D_{(k)}=\sqrt{b \delta \sum_{j=1}^{N}\left(B_{(k)(j)}\right)^{2}\left|\mathbf{x}_{(j)}-\mathbf{x}_{(k)}\right| V_{(j)}} .
$$

Therefore, the expression for yield function, $F_{(k)}^{[n+1]}$ can be rewritten as

$$
F_{(k)}^{[n+1]}=F_{(k)}^{[n+1]}\left(C_{(k)} ; s_{(k)(j)(t r i a l)}^{e[n+1]}, \bar{S}_{(k)}^{p[n]}\right)
$$

in which $C_{(k)}$ is the only unknown. Determination of $C_{(k)}$ which renders $F_{(k)}^{[n+1]}=0$ yields the magnitude of incremental plastic stretch, $\Delta s_{(k)(j)}^{p[n+1]}$. The search for this $C_{(k)}$ is achieved iteratively through Newton Raphson method as

$$
C_{(k)}^{n e w}=C_{(k)}^{o l d}-\frac{F_{(k)}^{[n+1]}\left(C_{(k)}^{o l d} ; S_{(k)(j)(t r i a l)}^{e[n+1]}, \bar{S}_{(k)}^{p[\mathrm{n}]}\right)}{\left.\frac{d F_{(k)}^{[n+1]}}{d C_{(k)}}\right|_{C_{(k)}=C_{(k)}^{o l d}}}
$$

(5) The magnitude of the plastic stretch increment, $C_{(k)}$ is determined once convergence is achieved, and the plastic stretch increment is calculated by using Eq. (28) as

$$
\Delta s_{(k)(j)}^{p[n+1]}=C_{(k)}\left(\frac{1}{2 \delta b}\left(t_{(k)(j)}\right)\left|\mathbf{x}_{(j)}-\mathbf{x}_{(k)}\right|+\frac{d}{b} a_{\mu}\left(1-\frac{a_{\kappa}}{a_{\mu}}\right) \theta_{(k)} \Lambda_{(k)(j)}\right) .
$$

(6) The plastic and elastic stretch components can be determined as

$$
S_{(k)(j)}^{\mathrm{p}[n+1]}=S_{(k)(j)}^{\mathrm{p}[n]}-\Delta S_{(k)(j)}^{\mathrm{p}[n+1]} .
$$

and

$S_{(k)(j)}^{\mathrm{e}[n+1]}=S_{(k)(j)}^{[n+1]}-S_{(k)(j)}^{\mathrm{p}[n+1]}$.

(7) Go to step (1).

\section{Numerical results}

An isotropic plate with and without defects in the form of a crack or a hole is considered in the generation of the numerical results. The square plate has an edge length of $L=1 \mathrm{~m}$. It has 
Young's modulus and Poisson's ratio of $E=113 \times 10^{9} \mathrm{~Pa}$ and $v=0.342$, respectively. For a linear isotropic hardening behavior, its yield stress and tangent modulus are specified as $\sigma_{y}=10.17 \times 10^{8} \mathrm{~Pa}$ and $K_{t}=1.38 \times 10^{9} \mathrm{~Pa}$, respectively. Also, its mass density is specified as $\rho=4428 \mathrm{~kg} / \mathrm{m}^{3}$.

\subsection{A plate under loading and unloading}

In order to demonstrate the validity of the present approach, the plate is subjected two loading paths. The first loading path involves only continuously increasing load beyond the yield stress. The second loading path involves loading, unloading, and loading again. The plate is on rollertype supports along the left end, i.e., $U^{*}=0$, and is subjected to a displacement gradient of $\varepsilon_{i}=\partial u / \partial x$ along the right end. During the first loading path, the applied displacement gradient increases linearly up to $\varepsilon_{i}=\partial u / \partial x=0.141$, as shown in Fig. 10a. The applied displacement gradient for the second loading path, with $\varepsilon_{i-2}=0.068, \varepsilon_{i-1}=0.0625$, and $\varepsilon_{i}=0.141$ is described in Fig. 10b. These boundary conditions are enforced as

$$
\begin{aligned}
& \begin{array}{l}
u_{f}\left(x_{f}, y_{f}, t+\Delta t\right)=2 U^{*}\left(x^{*}=0, y^{*}, t+\Delta t\right)-u(x, y, t) \\
v_{f}\left(x_{f}, y_{f}, t+\Delta t\right)=v(x, y, t)
\end{array} \text { with } x_{f} \in R_{f(x=0)}, \\
& \begin{array}{l}
u_{f}\left(x_{f}, y_{f}, t+\Delta t\right)=\varepsilon_{i}\left(x_{f}-x\right)+u(x, y, t) \\
v_{f}\left(x_{f}, y_{f}, t+\Delta t\right)=v(x, y, t)
\end{array} \text { with } x_{f} \in R_{f(x=L)} .
\end{aligned}
$$

The plate thickness is specified as $h=0.01 \mathrm{~m}$. It is discretized with a uniform grid of $100 \times 100$ material (integration) points.

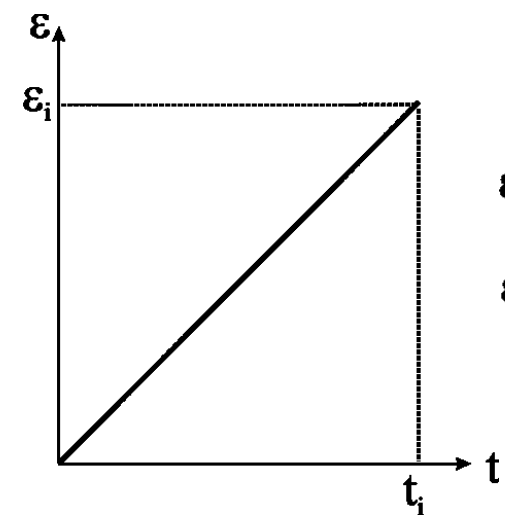

(a)

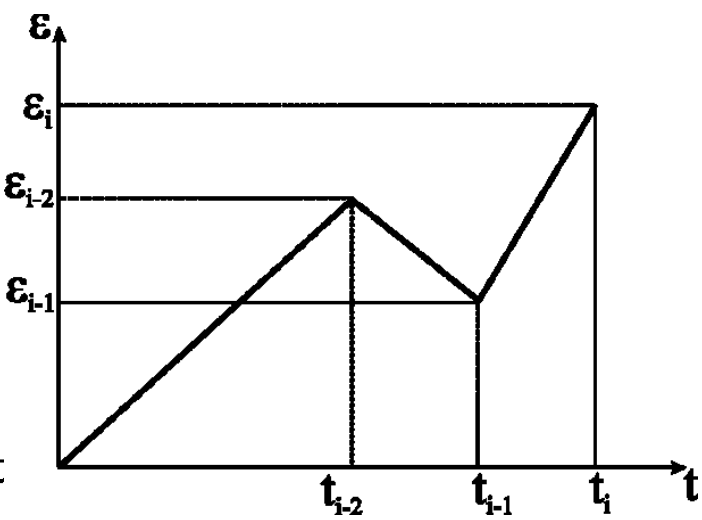

(b)

Fig. 10. Applied displacement gradients along the right edge of plate: (a) load path 1; (b) load path 2 .

As shown in Fig. 11, the PD predictions captures linear isotropic hardening for increasing load after the initial yielding. The PD prediction also captures the elastic unloading with a slope equal 
to the Young's modulus. During reloading plastic deformation continues to occur once the stress reaches the stress level where the unloading starts earlier as shown in Fig. 12

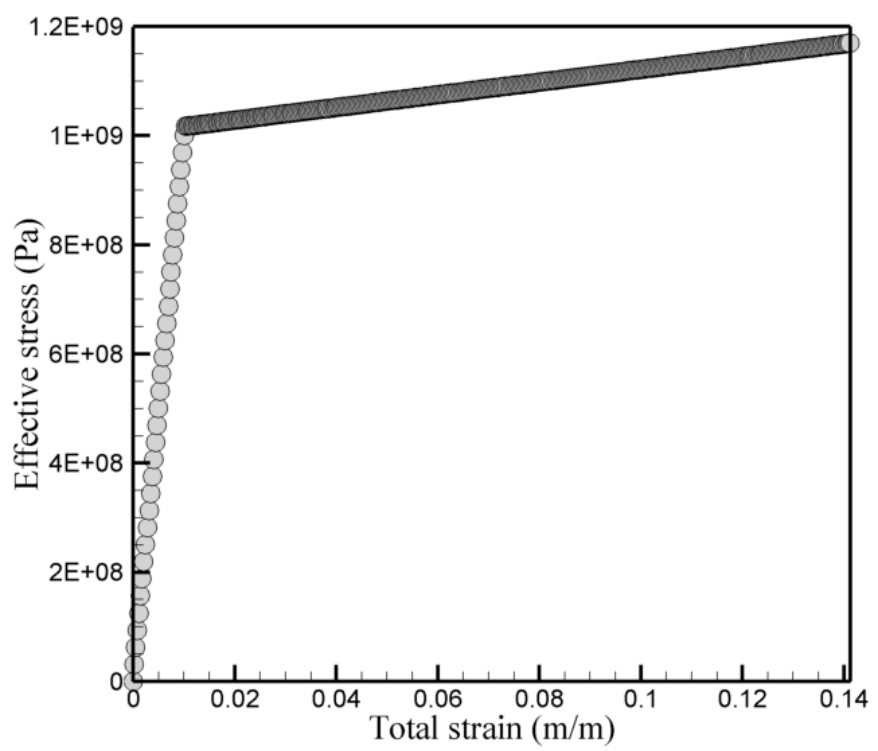

Fig 11. Variation of effective stress, $\bar{\sigma}_{e(k)}$, as the total stretch increases at the center of the plate: loading only.

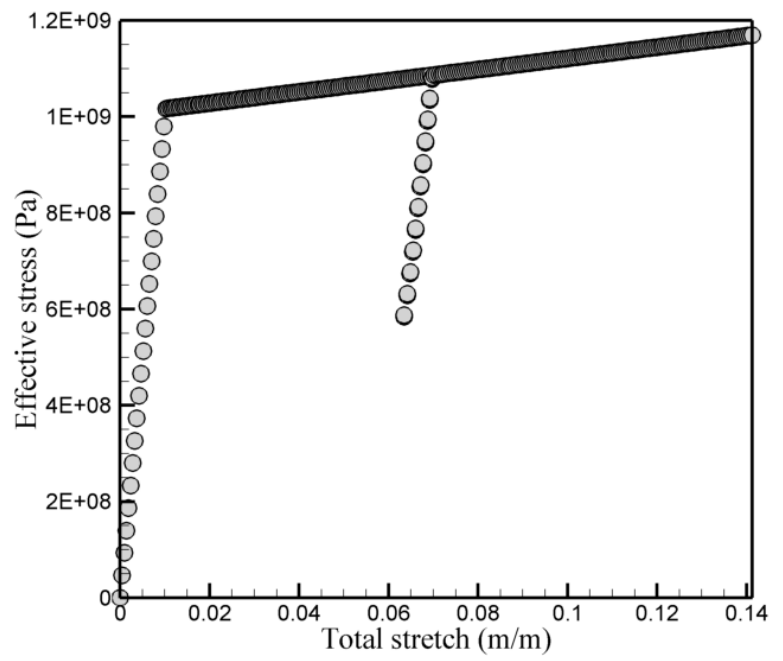

Fig 12. Variation of effective stress, $\bar{\sigma}_{e(k)}$, as the total stretch increases at the center of the plate: loading, unloading, and loading.

\subsection{Plate with a hole or a crack under tension}

The plate has either a central hole or a crack. The hole diameter and the crack length are specified as $D=0.3 \mathrm{~m}$. The plate is loaded symmetrically by applying displacement along the left 
and right ends. The applied displacement is increased linearly up to $u_{i}=0.005$, then decreased linearly to $u_{i}=0$. These boundary conditions are enforced as

$$
u_{f}\left(x_{f}, y_{f}, t+\Delta t\right)=2 u_{i}-u(x, y, t) \text { with } x_{f} \in R_{f(x=0)} \text {, }
$$

and

$u_{f}\left(x_{f}, y_{f}, t+\Delta t\right)=2 u_{i}-u(x, y, t)$ with $x_{f} \in R_{f(x=L)}$.

The plate geometry is discretized with a uniform $400 \times 400$ integration points. The thickness of the plate is specified as $h=0.0025 \mathrm{~m}$.

As expected, plastic deformation initiates in the regions of high stress concentrations. The size of the plastically deformed region expands as the loading increases. The variations of effective stress and equivalent plastic stretch for the case of the plate with a hole are presented in Figs. 1315. During loading, the size of the plastically deformed region becomes larger as observed in Figs. 13 and 14. However, the plastically deformed region remains unchanged during unloading as shown in Fig.15. The equivalent stretch distribution remains as a residual stretch. Upon unloading, the deformed geometry of the plate shown in Fig. 16 clearly captures the permanent change in the hole boundary.

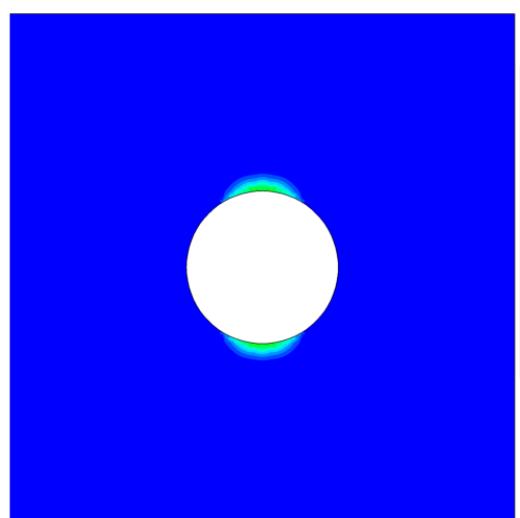

(a)

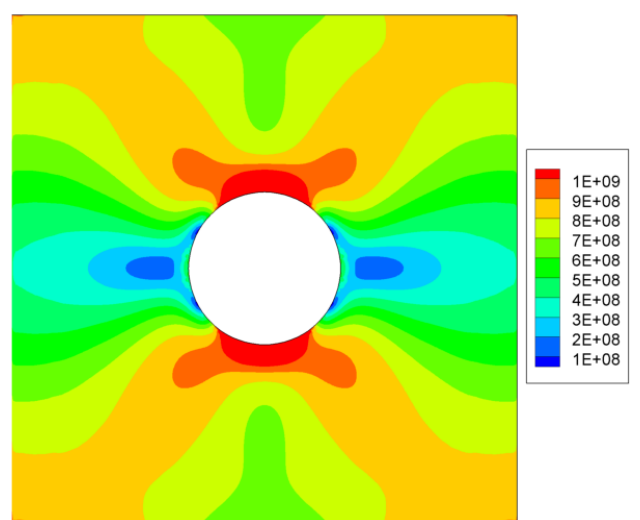

(b)

Fig. 13. Variations of (a) equivalent stretch and (b) effective stress in a plate with a hole due to loading of $u_{i}=0.004$. 


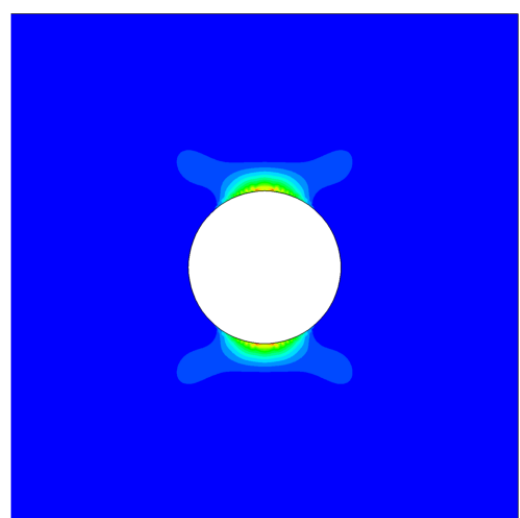

(a)

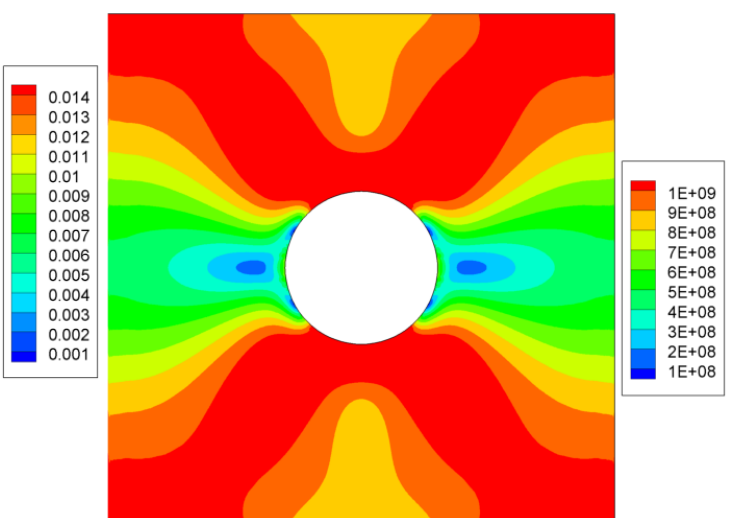

(b)

Fig. 14. Variations of (a) equivalent stretch and (b) effective stress in a plate with a hole due to loading of $u_{i}=0.005$.

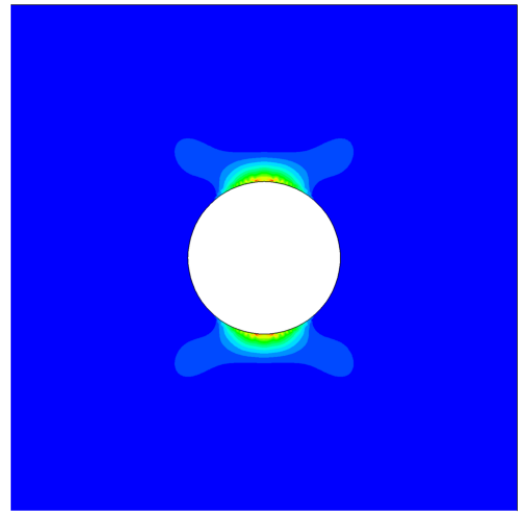

(a)

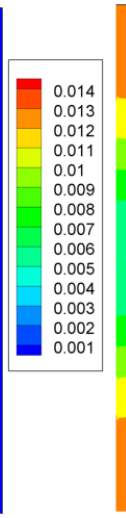

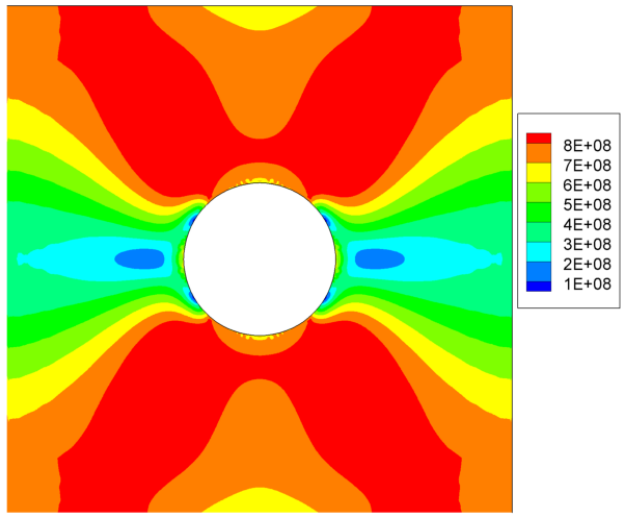

(b)

Fig. 15. Variations of (a) equivalent stretch and (b) effective stress in a plate with a hole due to loading of $u_{i}=0.005$ and unloading to $u_{i}=0.004$. 


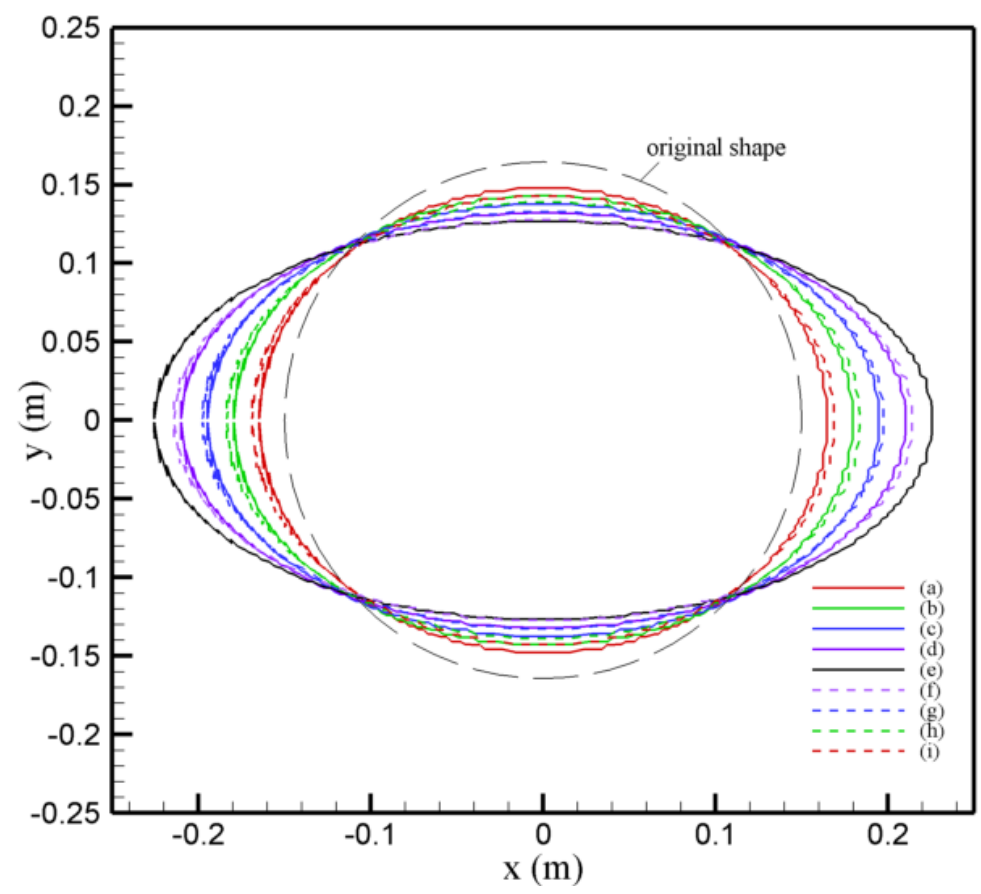

Fig. 16. PD predictions for change in hole shape due to applied loading conditions: Loading to (a) $u_{i}=0.001$; (b) $u_{i}=0.002$; (c) $u_{i}=0.003$; (d) $u_{i}=0.004$; (e) $u_{i}=0.005$; First loading to $u_{i}=0.005$ and then unloading to (f) $u_{i}=0.004$; (g) $u_{i}=0.003$; (h) $u_{i}=0.002$; (i) $u_{i}=0.001$ (displacements are magnified by 20 for the deformed configurations)

The variations of effective stress and equivalent plastic stretch for the case of the plate with a crack are presented in Figs. 17-19. The shape of the plastically deformed region has the same expected characteristic shape. As the loading increases, the size of the plastically deformed region increases. Upon unloading, the deformed geometry of the crack boundary shown in Fig. 20 clearly experiences permanent deformation. Also, the PD J-integral values are calculated according to $\mathrm{Hu}$ et al. [2012], and J-values are presented for increasing load in Fig. 21.

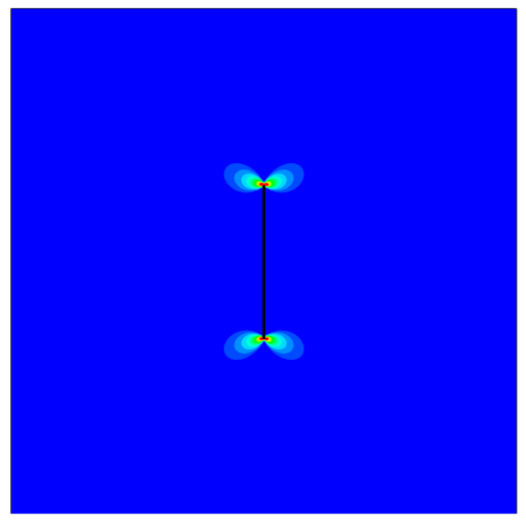

(a)

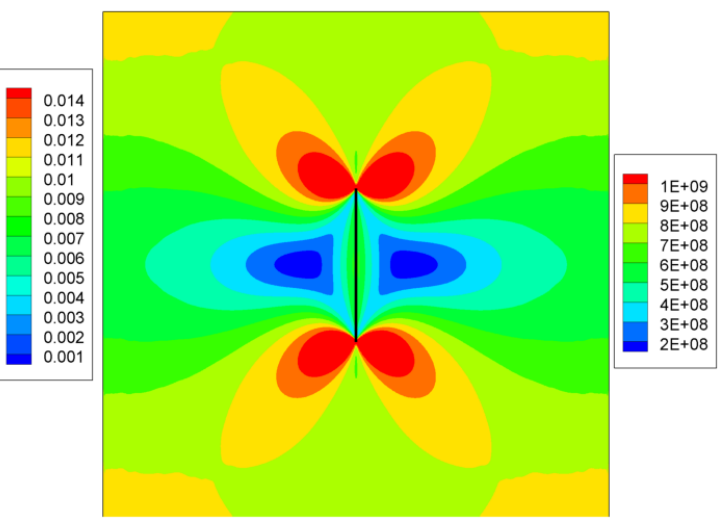

(b)

Fig. 17. Variations of (a) equivalent stretch and (b) effective stress in a plate with a crack due to loading of $u_{i}=0.004$. 


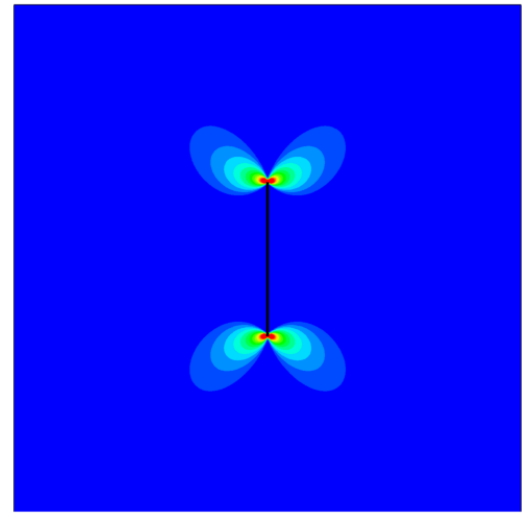

(a)

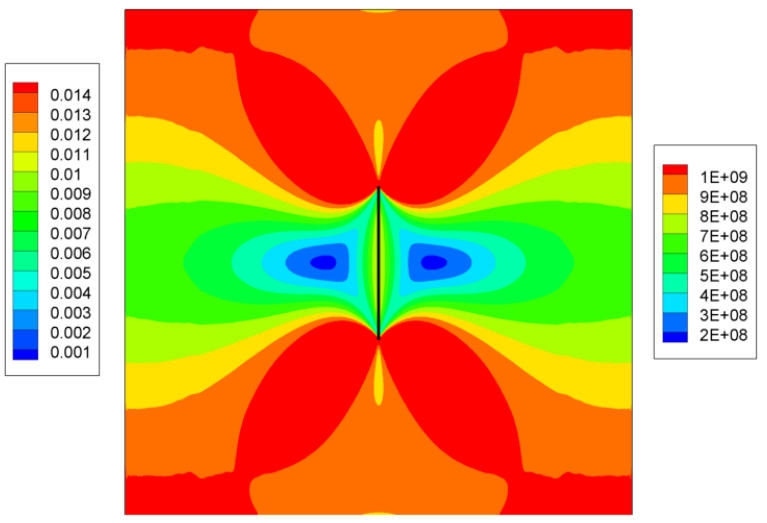

(b)

Fig. 18. Variations of (a) equivalent stretch and (b) effective stress in a plate with a crack due to loading of $u_{i}=0.005$.

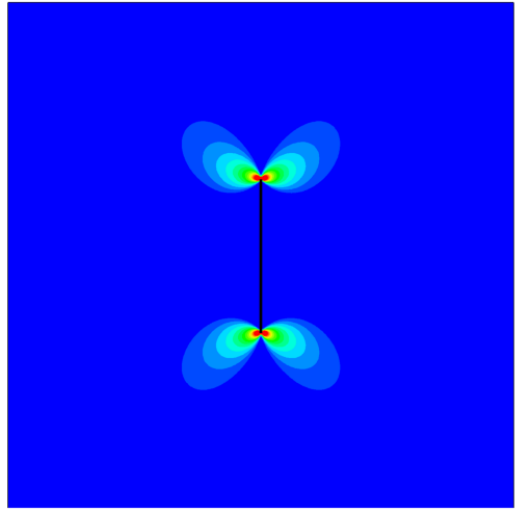

(a)

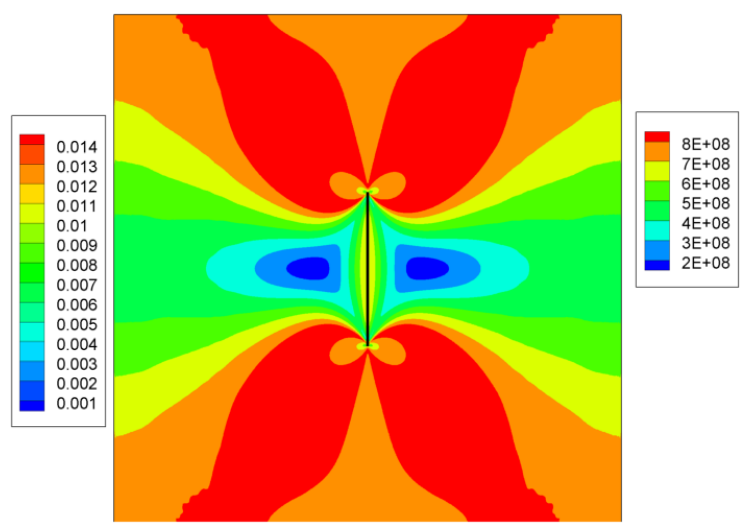

(b)

Fig. 19. Variations of (a) equivalent stretch and (b) effective stress in a plate with a crack due to loading of $u_{i}=0.005$ and unloading to $u_{i}=0.004$. 


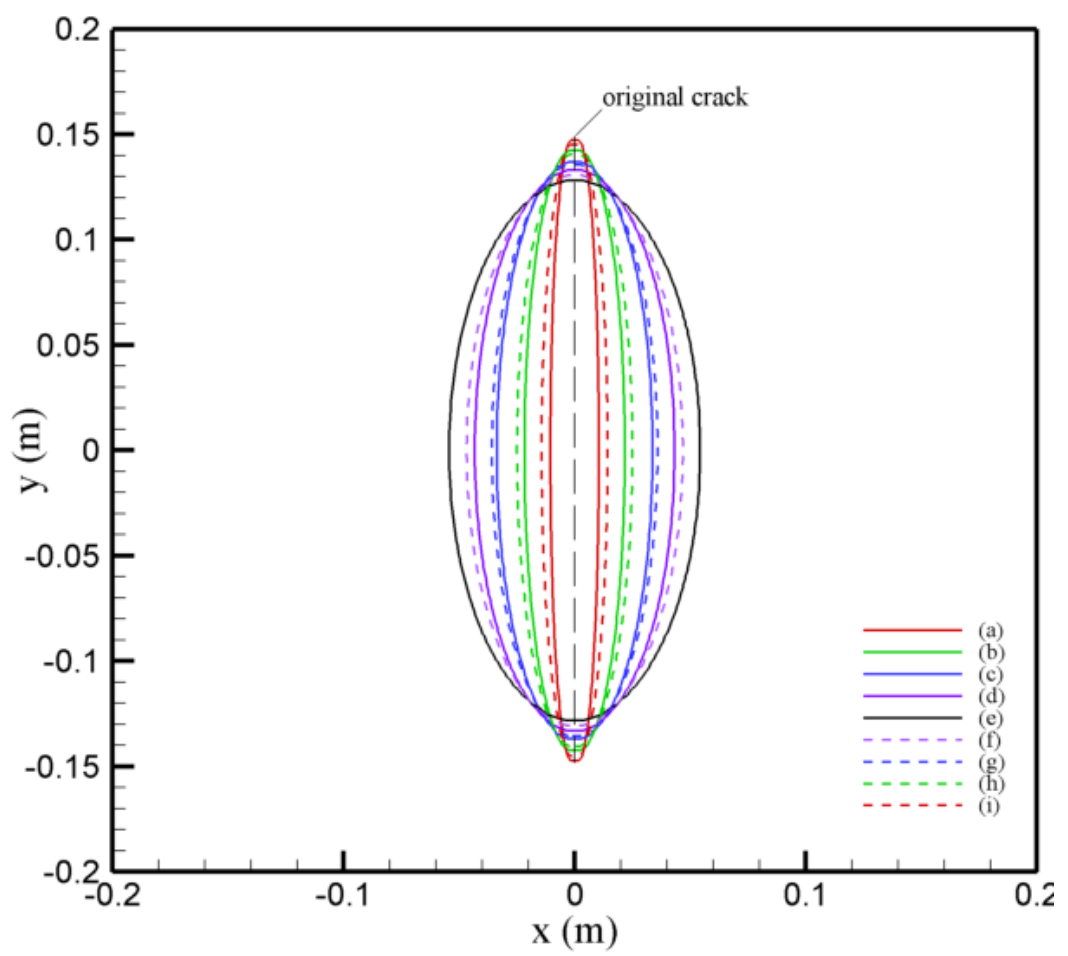

Fig. 20. PD predictions for change in crack shape due to applied loading conditions: Loading to (a) $u_{i}=0.001$; (b) $u_{i}=0.002$; (c) $u_{i}=0.003$; (d) $u_{i}=0.004$; (e) $u_{i}=0.005$; First loading to $u_{i}=0.005$ and then unloading to (f) $u_{i}=0.004$; (g) $u_{i}=0.003$; (h) $u_{i}=0.002$; (i) $u_{i}=0.001$ (displacements are magnified by 20 for the deformed configurations)

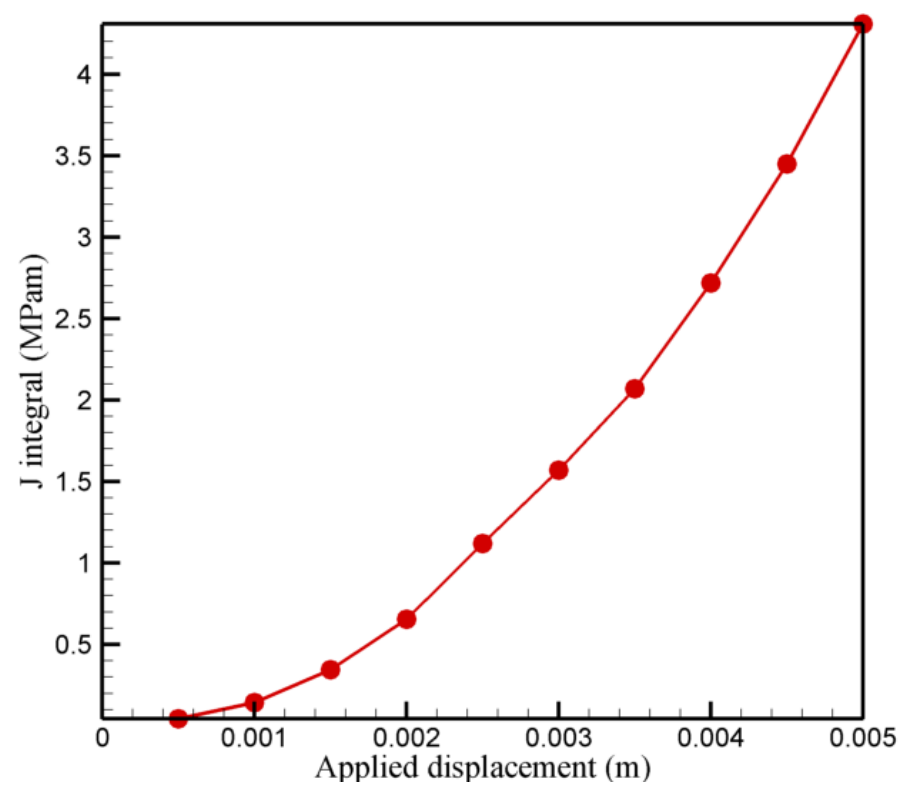

Fig. 21. Peridynamic J-integral values for increasing applied displacement 
According to the criteria given by Eq. (46), the crack is also allowed to propagate as the applied displacement is increased linearly up to $u_{i}=0.004$. The critical J-value for the material is specified as $J_{c}=3.4 \times 10^{7} \mathrm{~N} / \mathrm{m}$, i.e., $J_{(k)(j)}^{c}=1 \times 10^{6} \mathrm{~N} / \mathrm{m}$. Figure 22 shows the effective stress variations as the crack grows due to the increase in applied displacement. As expected, PD predictions yield a self-similar crack growth pattern. Also, the petal-shape plastic region moves with the crack tip as the crack propagates.

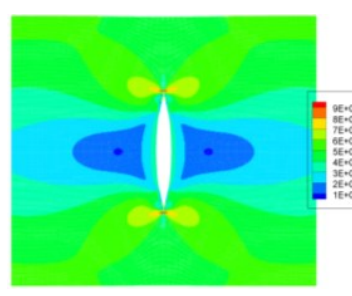

(a)

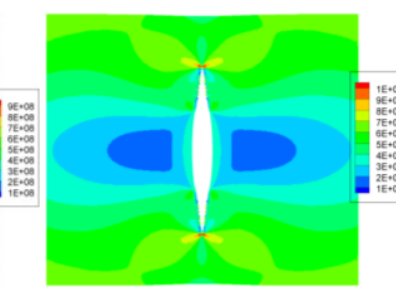

(b)

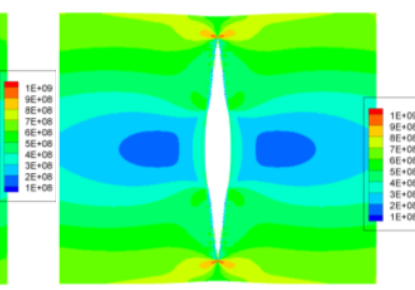

(c)

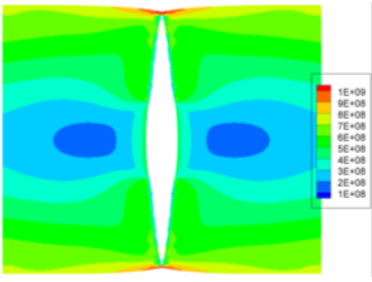

(d)

Fig. 22 . Effective stress variation as the crack grows under applied displacement with respect to the deformed configuration (magnified by 20): (a) $u_{i}=0.0025$; (b) $u_{i}=0.003$; (c) $u_{i}=0.0035$; (d) $u_{i}=0.004$.

\section{Concluding remarks}

This study presents the ordinary state-based peridynamic constitutive relations for plastic deformation based on von Mises yield criteria with isotropic hardening. Also, it presents a failure criteria based on the elastic-plastic micropotential of each interaction. The critical stretch critical energy release rate for each interaction is implicitly determined, and can vary depending on the degree of plastic deformation. The critical value to remove the interaction is related to the $J_{c^{-}}$ value of the material. The validity of peridynamic predictions is established by considering benchmark solutions concerning a plate under tension, a plate with a hole and a crack under tension.

\section{References}

Begley, J.A., Landes, J.D., 1972. The j-integral as a fracture criterion. Fracture Toughness. ASTM STP 514, American Society for Testing and Materials, pp. 1-23.

Breitenfeld, M.S., Geubelle, P.H., Weckner, O., Silling, S.A. , 2014, Non-ordinary state-based peridynamic analysis of stationary crack problems. Comput. Meth. Appl. Mech. Eng. 272, 233250

Drucker, D.C., 1951. A more fundamental approach to plastic stress-strain relations. Proceedings of the First U.S. National Congress of Applied Mechanics, ASME, pp. 487-491.

Foster, J.T., Silling, S.A., Chen, W., 2011, Viscoplasticity using peridynamics, Int. J Numer. Meth. Eng . 81, 1242-1258. 
Foster, J.T., Silling, S.A., Chen, W., 2011, An energy based failure criterion for use with peridynamic states, Int. J. Multiscale Comput. Eng. 9, 675-688.

Hu, W., Ha, Y.D., Bobaru, F., Silling, S.A., 2012. The formulation and computation of the nonlocal J-integral in bond-based peridynamics. Int. J. Fract. 176, 195-206.

Hu, Y., De Carvalho, N.V., Madenci, E., 2014. Peridynamic modeling of delamination growth in composite laminates. Compos. Struct. (submitted).

Kilic, B., Madenci, E., 2010. An adaptive dynamic relaxation method for quasi-static simulations using the peridynamic theory. Theor. Appl. Fract. Mech. 53, 194-201.

Macek, R.W., Silling, S.A., 2007. Peridynamics via finite element analysis. Finite Elem. Anal. Des. $43,1169-1178$.

Madenci, E., Oterkus, E., 2014. Peridynamic Theory and Its Applications. Springer, New York.

Mitchell, J.A., 2011. A nonlocal, ordinary, state-based plasticity model for peridynamics. SAND2011-3166, Sandia National Laboratories, Albuquerque.

Rice, J.R., 1968. A path independent integral and the approximate analysis of strain concentration by notches and cracks. J. Appl. Mech. 35, 379-386.

Silling, S.A., 2000. Reformulation of elasticity theory for discontinuities and long-range forces. J. Mech. Phys. Solids 48, 175-209.

Silling, S.A., Askari, E., 2005. A meshfree method based on the peridynamic model of solid mechanics. Comput. Struct. 83, 526-1535.

Silling, S.A., Epton, M., Weckner, O., Xu, J., Askari, A., 2007. Peridynamics states and constitutive modeling. J. Elast. 88, 151-184.

Silling, S.A., Lehoucq, R.B., 2008, Convergence of peridynamics to classical elasticity theory. J. Elast. 93, 13-37.

Silling, S.A., Lehoucq, R.B., 2010. Peridynamic theory of solid mechanics. In: Aref, H., van der Giessen, H. (Eds.), Advances in Applied Mechanics. Elsevier, San Diego, pp. 73-168.

Taylor, M.J., 2008. Numerical simulation of thermo-elasticity, inelasticity and rupture in membrane theory. Dissertation, University of California, Berkeley 


\section{Appendix A}

The correction of the material parameters is achieved by numerically integrating both dilatation and strain energy density at each material point inside the body for simple loading conditions and comparing them to their counterparts obtained from classical continuum mechanics. The correction factor can be calculated by considering simple loading conditions as suggested by Madenci and Oterkus (2014).

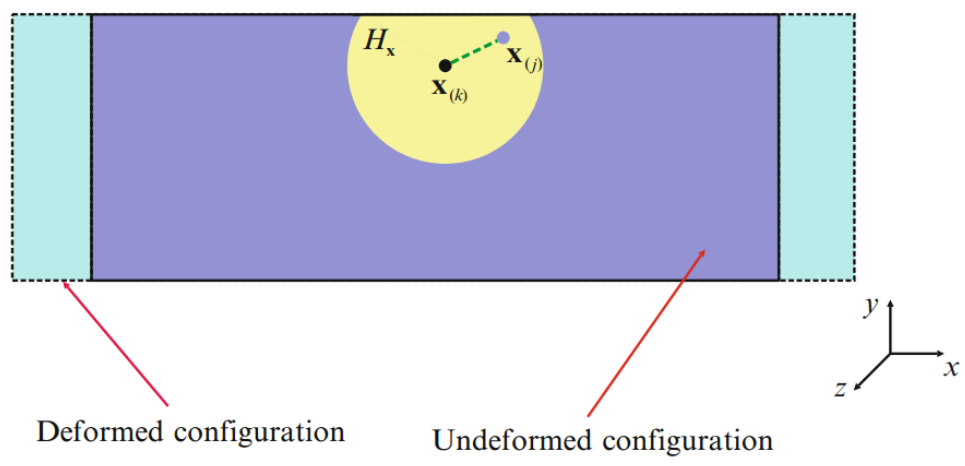

Fig. A.1. Peridynamic plate subjected to uniaxial stretch in the $x$-direction.

For a two-dimensional analysis, the simple loading conditions are achieved by applying uniaxial stretch in the $x$ - and $y$-directions of the coordinate system, i.e., $\varepsilon_{x x} \neq 0, \varepsilon_{\alpha a}=\gamma_{\alpha \beta}=0$ (shown in Fig. A.1) and $\varepsilon_{y y} \neq 0, \varepsilon_{\alpha a}=\gamma_{\alpha \beta}=0$, respectively, with $\alpha, \beta=x, y$. This is achieved through the constant displacement gradient, $\partial u_{\alpha}^{*} / \partial \alpha=\zeta$, with $\alpha=x, y$. The displacement field at material point $\mathbf{x}$ resulting from this loading can be expressed as

$$
\mathbf{u}_{1}^{T}(\mathbf{x})=\left\{\begin{array}{lll}
\frac{\partial u_{x}^{*}}{\partial x} x & 0 & 0
\end{array}\right\},
$$

and

$$
\mathbf{u}_{2}^{T}(\mathbf{x})=\left\{\begin{array}{lll}
0 & \frac{\partial u_{y}^{*}}{\partial y} y & 0
\end{array}\right\},
$$

in which the subscripts $(1,2)$ denote the $x$ - and $y$-directions of uniaxial stretch, respectively. Due to this displacement field, the corresponding PD dilatation term, $\theta_{m}^{P D}\left(\mathbf{x}_{(k)}\right)$ with $(m=1,2)$, at material point $\mathbf{x}_{(k)}$ can be obtained from Eq. (3a) as

$$
\theta_{m}^{P D}\left(\mathbf{x}_{(k)}\right)=d \delta \sum_{j=1}^{N} s_{(k)(j)} \Lambda_{(k)(j)} V_{(j)}
$$


in which $N$ represents the number of material points inside the horizon of material point $\mathbf{x}_{(k)}$. The corresponding dilatation based on classical continuum mechanics, $\theta_{m}^{C M}\left(\mathbf{x}_{(k)}\right)$, is uniform throughout the domain and is determined as

$$
\theta_{m}^{C M}\left(\mathbf{x}_{(k)}\right)=\zeta
$$

The dilatation correction term can be defined as

$$
D_{m(k)}=\frac{\theta_{m}^{C M}\left(\mathbf{x}_{(k)}\right)}{\theta_{m}^{P D}\left(\mathbf{x}_{(k)}\right)}=\frac{\zeta}{d \delta \sum_{j=1}^{N} s_{(k)(j)} \Lambda_{(k)(j)} V_{(j)}} .
$$

Due to these applied displacement fields, the PD strain energy density at material point $\mathbf{x}_{(k)}$ can be obtained from Eq. (2a) as

$$
W_{m}^{P D}\left(\mathbf{x}_{(k)}\right)=a\left(\theta_{m}^{P D}\left(\mathbf{x}_{(k)}\right)\right)^{2}+b \delta \sum_{j=1}^{N} \frac{1}{\left|\mathbf{x}_{(j)}-\mathbf{x}_{(k)}\right|}\left(\left|\mathbf{y}_{(j)}-\mathbf{y}_{(k)}\right|-\left|\mathbf{x}_{(j)}-\mathbf{x}_{(k)}\right|\right)^{2} V_{(j)}
$$

with $(m=1,2)$ and $a=a_{\kappa}-a_{\mu}$. With the corrected dilatation, this expression can be rewritten as results in

$$
W_{m}^{P D}\left(\mathbf{x}_{(k)}\right)-a \zeta^{2}=b \delta \sum_{j=1}^{N} \frac{1}{\left|\mathbf{x}_{(j)}-\mathbf{x}_{(k)}\right|}\left(\left|\mathbf{y}_{(j)}-\mathbf{y}_{(k)}\right|-\left|\mathbf{x}_{(j)}-\mathbf{x}_{(k)}\right|\right)^{2} V_{(j)} .
$$

Hence, the correction term can be defined as

$$
S_{m(k)}=\frac{W_{(m)}^{C M}\left(\mathbf{x}_{(k)}\right)-a \zeta^{2}}{W_{(m)}^{P D}\left(\mathbf{x}_{(k)}\right)-a \zeta^{2}}=\frac{\frac{1}{2\left(1-v^{2}\right)} E \zeta^{2}-a \zeta^{2}}{b \delta \sum_{j=1}^{N} \frac{1}{\left|\mathbf{x}_{(j)}-\mathbf{x}_{(k)}\right|}\left(\left|\mathbf{y}_{(j)}-\mathbf{y}_{(k)}\right|-\left|\mathbf{x}_{(j)}-\mathbf{x}_{(k)}\right|\right)^{2} V_{(j)}} .
$$

These corrections are valid only in the principle directions. The surface correction for any direction can be obtained by assuming a variation in the form of an ellipse, as explained in Madenci and Oterkus (2014).

With these expressions, a vector of correction factors for the integral terms in dilatation and strain energy density at material point $\mathbf{x}_{(k)}$ can be written as

$$
\mathbf{g}_{(d)}\left(\mathbf{x}_{(k)}\right)=\left\{g_{x(d)(k)}, g_{y(d)(k)}\right\}^{T}=\left\{D_{1(k)}, D_{2(k)}\right\}^{T}
$$




$$
\mathbf{g}_{(b)}\left(\mathbf{x}_{(k)}\right)=\left\{g_{x(b)(k)}, g_{y(b)(k)}\right\}^{T}=\left\{S_{1(k)}, S_{2(k)}\right\}^{T}
$$

These correction factors are only valid in the $x$ - and $y$-directions. However, they can be used as the principal values of an ellipse, as shown in Fig. A.2, in order to approximate the surface correction factor in any direction. Arising from a general loading condition, the correction factor for interaction between material points $\mathbf{x}_{(k)}$ and $\mathbf{x}_{(j)}$, shown in Fig. A.2, can be obtained in the direction of their unit relative position vector, $\mathbf{n}=\left(\mathbf{x}_{(j)}-\mathbf{x}_{(k)}\right) /\left|\mathbf{x}_{(j)}-\mathbf{x}_{(k)}\right|=\left\{n_{x}, n_{y}\right\}^{T}$.

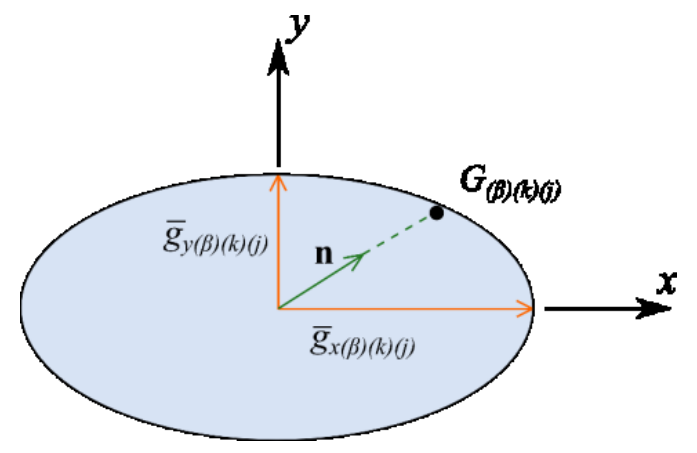

Fig. A.2. The ellipse for the surface corrections.

A vector of correction factors for the integrals in the dilatation and strain energy density expressions at material point $\mathbf{x}_{(j)}$ can be similarly written as

$$
\begin{aligned}
& \mathbf{g}_{(d)(j)}\left(\mathbf{x}_{(j)}\right)=\left\{g_{x(d)(j)}, g_{y(d)(j)}\right\}^{T}=\left\{D_{1(j)}, D_{2(j)}\right\}^{T}, \\
& \mathbf{g}_{(b)(j)}\left(\mathbf{x}_{(j)}\right)=\left\{g_{x(b)(j)}, g_{y(b)(j)}\right\}^{T}=\left\{S_{1(j)}, S_{2(j)}\right\}^{T} .
\end{aligned}
$$

These correction factors are, in general, different at material points $\mathbf{x}_{(k)}$ and $\mathbf{x}_{(j)}$. Therefore, the correction factor for an interaction between material points $\mathbf{x}_{(k)}$ and $\mathbf{x}_{(j)}$ can be obtained by their mean values as

$$
\overline{\mathbf{g}}_{(\beta)(k)(j)}=\left\{\bar{g}_{x(\beta)(k)(j)}, \bar{g}_{y(\beta)(k)(j)}\right\}^{T}=\frac{\mathbf{g}_{(\beta)(k)}+\mathbf{g}_{(\beta)(j)}}{2},
$$

with $\beta=d, b$, which can be used as the principal values of an ellipse, as shown in Fig. A.2.

The intersection of the ellipsoid and a relative position vector, $\mathbf{n}=\left(\mathbf{x}_{(j)}-\mathbf{x}_{(k)}\right) /\left|\mathbf{x}_{(j)}-\mathbf{x}_{(k)}\right|$, of material points $\mathbf{x}_{(k)}$ and $\mathbf{x}_{(j)}$ provides the correction factors as 


$$
G_{(\beta)(k)(j)}=\left(\left[n_{x} / \bar{g}_{x(\beta)(k)(j)}\right]^{2}+\left[n_{y} / \bar{g}_{y(\beta)(k)(j)}\right]^{2}\right)^{-1 / 2} .
$$

After considering the surface effects, the discrete forms of the dilatation and the strain energy density can be corrected as

$$
\begin{aligned}
& \theta_{(k)}=d \delta \sum_{j=1}^{N} G_{(d)(k)(j)} S_{(k)(j)} \Lambda_{(k)(j)} V_{(j)}, \\
& W_{(k)}=a \theta_{(k)}^{2}+b \delta \sum_{j=1}^{N} G_{(b)(k)(j)} \frac{1}{\left|\mathbf{x}_{(j)}-\mathbf{x}_{(k)}\right|}\left(\left|\mathbf{y}_{(j)}-\mathbf{y}_{(k)}\right|-\left|\mathbf{x}_{(j)}-\mathbf{x}_{(k)}\right|\right)^{2} V_{(j)} .
\end{aligned}
$$

Although this method enables the correction to material parameters due to the presence of a surface or an interface, it is still a numerical approximation.

\section{Appendix B}

The parameter $A_{0}$ in Eq. (30) is determined in such a way that $\Delta \bar{s}_{(k)}^{p}$ recovers the incremental plastic stretch due to uniaxial tension. In three-dimensional analysis, the incremental plastic strains are $\Delta \varepsilon_{x x(k)}^{p}=\Delta \zeta$ and $\Delta \varepsilon_{y y(k)}^{p}=\Delta \varepsilon_{z z(k)}^{p}=-\Delta \zeta / 2$ (arising from zero dilatation due to plastic deformation) and $\gamma_{x y(k)}^{p}=\gamma_{x z(k)}^{p}=\gamma_{y z(k)}^{p}=0$ under incremental uniaxial tensile loading. The distortional part of strain energy density due to this incremental plastic stretch, $\Delta \zeta$, can be computed by evaluating

$$
W_{(k)}^{\mu}\left(d s_{(k)(j)}^{p}\right)=b \sum_{j=1}^{N} w_{(k)(j)}\left(\left|\mathbf{y}_{(j)}-\mathbf{y}_{(k)}\right|-\left|\mathbf{x}_{(j)}-\mathbf{x}_{(k)}\right|\right)^{2} V_{(j)} .
$$

In reference to the Cartesian and spherical coordinates, the relative position vector in the undeformed and deformed states between material points can be expressed as

$$
\mathbf{x}_{(j)}-\mathbf{x}_{(k)}=\boldsymbol{\xi}=\xi_{x} \boldsymbol{i}+\xi_{y} \boldsymbol{j}+\xi_{z} \boldsymbol{k}=\boldsymbol{\xi} \mathbf{e}_{r}+\xi \mathbf{e}_{\theta}+\xi \mathbf{e}_{\phi},
$$

with

$$
\xi_{x}=\xi \sin \phi \cos \theta ; \quad \xi_{y}=\xi \sin \phi \sin \theta ; \quad \xi_{z}=\xi \cos \phi
$$

and

$$
\mathbf{y}_{(j)}-\mathbf{y}_{(k)}=A_{x} \boldsymbol{i}+\boldsymbol{A}_{y} \boldsymbol{j}+\boldsymbol{A}_{z} \boldsymbol{k}=A_{r} \mathbf{e}_{r}+A_{\theta} \mathbf{e}_{\theta}+A_{\phi} \mathbf{e}_{\phi} .
$$


The relative displacement vector between these points is

$$
\left(\mathbf{y}_{(j)}-\mathbf{y}_{(k)}\right)-\left(\mathbf{x}_{(j)}-\mathbf{x}_{(k)}\right)=\left(A_{x}-\xi_{x}\right) \boldsymbol{i}+\left(A_{y}-\xi_{y}\right) \boldsymbol{j}+\left(A_{z}-\xi_{z}\right) \boldsymbol{k},
$$

or

$$
\left(\mathbf{y}_{(j)}-\mathbf{y}_{(k)}\right)-\left(\mathbf{x}_{(j)}-\mathbf{x}_{(k)}\right)=\left(A_{r}-\xi\right) \mathbf{e}_{r}+\left(A_{\theta}-\xi\right) \mathbf{e}_{\theta}+\left(A_{\phi}-\xi\right) \mathbf{e}_{\phi} .
$$

Due to the deformation arising from incremental strains, the coefficients of the corresponding relative position vector can be expressed as

$$
\left\{\begin{array}{l}
A_{x} \\
A_{y} \\
A_{z}
\end{array}\right\}=\left\{\begin{array}{l}
(1+\Delta \zeta) \xi_{x} \\
\left(1-\frac{\Delta \zeta}{2}\right) \xi_{y} \\
\left(1-\frac{\Delta \zeta}{2}\right) \xi_{z}
\end{array}\right\}
$$

In accordance with Fig. B.1, the components of this vector can be expressed in spherical coordinates through the transformation given by

$$
\left\{\begin{array}{l}
A_{r} \\
A_{\phi} \\
A_{\theta}
\end{array}\right\}=\left[\begin{array}{ccc}
\sin (\phi) \cos (\theta) & \sin (\phi) \sin (\theta) & \cos (\phi) \\
-\cos (\phi) \cos (\theta) & \cos (\phi) \sin (\theta) & -\sin (\phi) \\
-\sin (\theta) & \cos (\theta) & 0
\end{array}\right]\left\{\begin{array}{l}
A_{x} \\
A_{y} \\
A_{z}
\end{array}\right\} .
$$

In particular, the radial component becomes

$$
A_{r}=(1+\Delta \zeta) \xi \sin ^{2} \phi \cos ^{2} \theta+\left(1-\frac{\Delta \zeta}{2}\right) \xi \sin ^{2} \phi \sin ^{2} \theta+\left(1-\frac{\Delta \zeta}{2}\right) \xi \cos ^{2}(\phi) .
$$

This expression can be further simplified as

$$
A_{r}=\left[1+\frac{3}{2} \Delta \zeta \sin ^{2} \phi \cos ^{2} \theta-\frac{\Delta \zeta}{2}\right] \xi
$$

For this incremental deformation, the distance between the material points $\mathbf{x}_{(j)}$ and $\mathbf{x}_{(k)}$ in the deformed configuration is

$$
\left|\mathbf{y}_{(j)}-\mathbf{y}_{(k)}\right|=\left[1+\frac{3 \Delta \zeta}{2} \sin ^{2}(\phi) \cos ^{2}(\theta)-\frac{\Delta \zeta}{2}\right]\left|\mathbf{x}_{(j)}-\mathbf{x}_{(k)}\right| .
$$


Therefore, the incremental plastic strain energy, $\Delta W_{(k)}^{p}$, due to incremental uniaxial plastic stretch, $\Delta \zeta$, can be evaluated as

$$
\Delta W_{(k)}^{\mu}=b \int_{0}^{\delta} \int_{0}^{2 \pi} \int_{0}^{\pi} \frac{\delta}{\xi}\left(\left[1+\frac{3 \Delta \zeta}{2} \sin ^{2}(\phi) \cos ^{2}(\theta)-\frac{\Delta \zeta}{2}\right] \xi-\xi\right)^{2} \xi^{2} \sin (\phi) d \phi d \theta d \xi
$$

or

$$
\Delta W_{(k)}^{\mu}=\frac{\pi}{5} b \delta^{5}(\Delta \zeta)^{2}
$$

Requiring that the equivalent incremental plastic stretch, $\Delta \bar{s}_{(k)}^{p}$, recover the incremental plastic strain, $\Delta \zeta$, due to uniaxial tension leads to

$$
A_{0}=\frac{\sqrt{5}}{\sqrt{\pi b \delta^{5}}}
$$

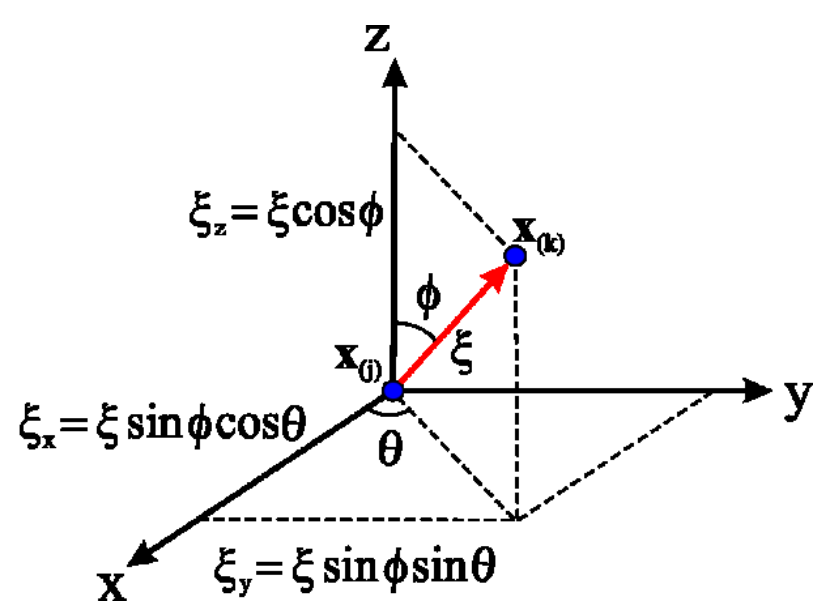

Fig. B.1. Spherical coordinates.

For two-dimensional analysis under uniaxial tension, the incremental plastic strains are $\Delta \varepsilon_{x x(k)}^{p}=\Delta \zeta$ and $\Delta \varepsilon_{y y(k)}^{p}=-\Delta \zeta$ (arising from zero dilatation due to plastic deformation) and $\gamma_{x y(k)}^{p}=0$ under incremental uniaxial tensile loading. The corresponding relative position vector in the undeformed and deformed state between material points can be expressed as

$$
\mathbf{x}_{(j)}-\mathbf{x}_{(k)}=\boldsymbol{\xi}=\xi_{x} \boldsymbol{i}+\xi_{y} \boldsymbol{j}=\xi_{\mathrm{e}_{r}}+\xi_{\mathbf{e}_{\theta}}
$$

with

$$
\xi_{x}=\xi \cos \theta ; \quad \xi_{y}=\xi \sin \theta
$$


and

$$
\mathbf{y}_{(j)}-\mathbf{y}_{(k)}=A_{x} \boldsymbol{i}+\boldsymbol{A}_{y} \boldsymbol{j}=A_{r} \mathbf{e}_{r}+A_{\theta} \mathbf{e}_{\theta}
$$

The relative displacement vector between these points can be expressed as

$$
\left(\mathbf{y}_{(j)}-\mathbf{y}_{(k)}\right)-\left(\mathbf{x}_{(j)}-\mathbf{x}_{(k)}\right)=\left(A_{x}-\xi_{x}\right) \boldsymbol{i}+\left(A_{y}-\xi_{y}\right) \boldsymbol{j}
$$

or

$$
\left(\mathbf{y}_{(j)}-\mathbf{y}_{(k)}\right)-\left(\mathbf{x}_{(j)}-\mathbf{x}_{(k)}\right)=\left(A_{r}-\xi\right) \mathbf{e}_{r}+\left(A_{\theta}-\xi\right) \mathbf{e}_{\theta}
$$

Due to the deformation arising from incremental strains, the coefficients of the corresponding relative position vector can be expressed as

$$
\left\{\begin{array}{l}
A_{x} \\
A_{y}
\end{array}\right\}=\left\{\begin{array}{l}
(1+\Delta \zeta) \xi_{x} \\
(1-\Delta \zeta) \xi_{y}
\end{array}\right\}
$$

Using the coordinate transformation

$$
\left\{\begin{array}{l}
A_{r} \\
A_{\theta}
\end{array}\right\}=\left[\begin{array}{cc}
\cos (\theta) & \sin (\theta) \\
-\sin (\theta) & \cos (\theta)
\end{array}\right]\left\{\begin{array}{c}
A_{x} \\
A_{y}
\end{array}\right\}
$$

The radial component becomes

$$
A_{r}=(1+\Delta \zeta) \xi \cos ^{2} \theta+(1-\Delta \zeta) \xi \sin ^{2}(\theta)
$$

Therefore, the incremental plastic strain energy, $\Delta W_{(k)}^{p}$, due to incremental uniaxial plastic stretch, $\Delta \zeta$ can be evaluated as

$$
\Delta W_{(k)}^{\mu}=b h \int_{0}^{\delta} \int_{0}^{2 \pi} \frac{\delta}{\xi}\left(\left[(1+\Delta \zeta) \cos ^{2}(\theta)+(1-\Delta \zeta) \sin ^{2}(\theta)\right] \xi-\xi\right)^{2} \xi d \theta d \xi
$$

or

$$
\Delta W_{(k)}^{\mu}=\frac{1}{3} b h \Delta \zeta^{2} \delta^{4} \pi
$$

Requiring that the equivalent incremental plastic stretch,,$\Delta \bar{s}_{(k)}^{p}$, recover the incremental plastic strain, $\Delta \zeta$, due to uniaxial tension leads to 


$$
A_{0}=\frac{\sqrt{3}}{\sqrt{\pi b h \delta^{4}}}
$$

For one-dimensional analysis under uniaxial tension, the incremental plastic strain is $\Delta \varepsilon_{x x(k)}^{p}=\Delta \zeta$ . For this deformation, the deformed configuration of the distance between the material points $\mathbf{x}_{(j)}$ and $\mathbf{x}_{(k)}$ is

$$
\left|\mathbf{y}_{(j)}-\mathbf{y}_{(k)}\right|=(1+\Delta \zeta)\left|\mathbf{x}_{(j)}-\mathbf{x}_{(k)}\right|
$$

Therefore, the incremental plastic strain energy, $\Delta W_{(k)}^{p}$, due to incremental uniaxial plastic stretch, $\Delta \zeta$, can be evaluated as

$$
\Delta W_{(k)}^{\mu}=2 A \int_{0}^{\delta} b \delta(\Delta \zeta)^{2} \xi \mathrm{d} \xi=A b \delta^{3}(\Delta \zeta)^{2}
$$

Requiring that the equivalent incremental plastic stretch, $\Delta \bar{s}_{(k)}^{p}$, recover the incremental plastic strain, $\Delta \zeta$, due to uniaxial tension leads to

$$
A_{0}=\frac{1}{\sqrt{A b \delta^{3}}}
$$

\section{Appendix C}

The validity of the approach for imposing different types of boundary conditions is demonstrated by considering the elastic deformation of a rectangular plate first subjected to nonzero displacement boundary constraints, and then to a combination of zero displacement constraints and traction boundary conditions.

\section{C.1. Displacement constraint conditions}

As shown in Fig. C.1, a rectangular plate is subjected to displacement constraints of $u(x=0, y, t)=U^{*}=-0.0005 \mathrm{~m}$ and $u(x=L, y, t)=U^{*}=0.0005 \mathrm{~m}$. It is free of tractions. The plate has a length of $L=1 \mathrm{~m}$, width of $H=0.5 \mathrm{~m}$, and thickness of $h=0.01 \mathrm{~m}$. Its Young's modulus and Poisson's ratio are specified as $E=200 \times 10^{9} \mathrm{~Pa}$ and $v=1 / 3$, respectively, with mass density $\rho=7850 \mathrm{~kg} / \mathrm{m}^{3}$. The boundary conditions are enforced as

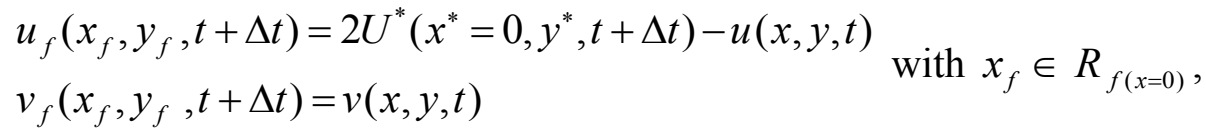




$$
\begin{aligned}
& u_{f}\left(x_{f}, y_{f}, t+\Delta t\right)=2 U^{*}\left(x^{*}=L, y^{*}, t+\Delta t\right)-u(x, y, t) \\
& v_{f}\left(x_{f}, y_{f}, t+\Delta t\right)=v(x, y, t)
\end{aligned} \text { with } x_{f} \in R_{f(x=L)} .
$$

The PD model is constructed by considering 100 and 50 material points in the $x$ - and $y$-directions, respectively. It results in a uniform spacing of $\Delta=1 \times 10^{-2} \mathrm{~m}$ between material points.

Also, the horizon is specified as $\delta=3.015 \Delta$. The PD displacement predictions are shown in Fig C.2. As expected, the plate elongates uniformly in the axial direction and contracts in the longitudinal direction.

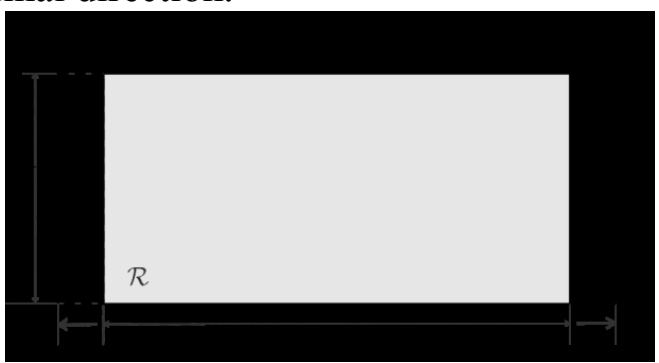

(a)

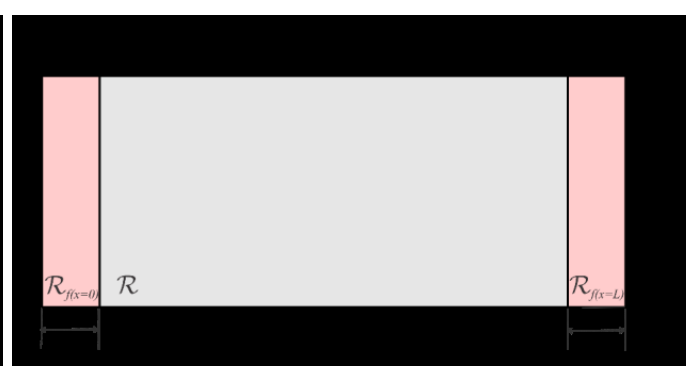

(b)

Fig. C.1. Rectangular plate under uniform stretch: (a) geometry and loading; (b) PD model with fictitious regions.

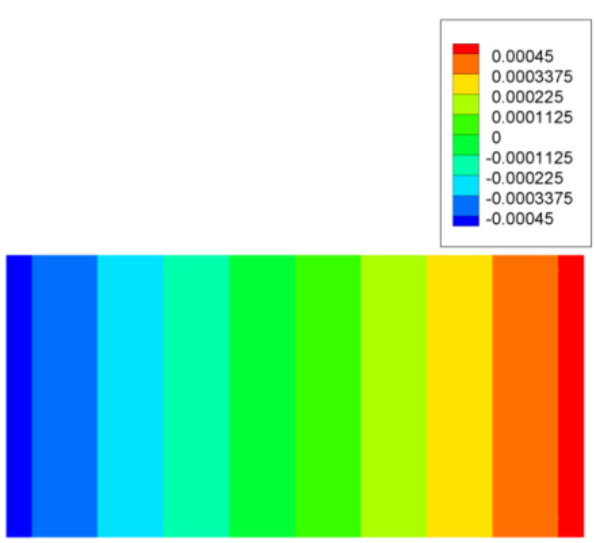

(a)
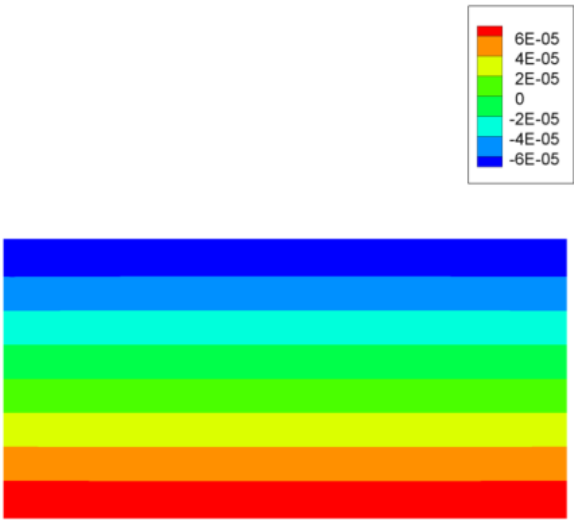

(b)

Fig. C.2 PD solutions for a) Horizontal displacement, and b) Vertical displacement

\section{C.2. Combined displacement constraint and traction conditions}

The rectangular plate is subjected to a uniform normal stress of $\sigma_{0}=100 \times 10^{6} \mathrm{~Pa}$ on the right side and is fixed on the left side, $u(x=0, y, t)=0$ and $v(x=0, y, t)=0$, as shown in Fig. C.3. 


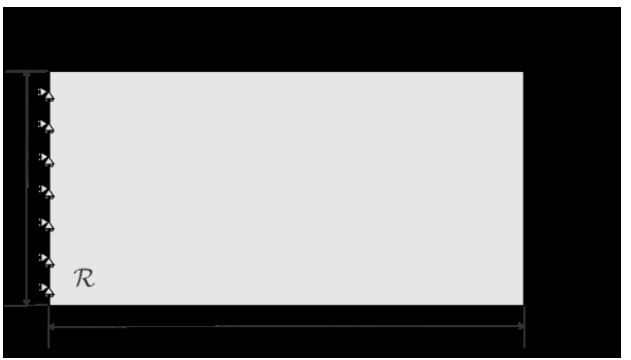

(a)

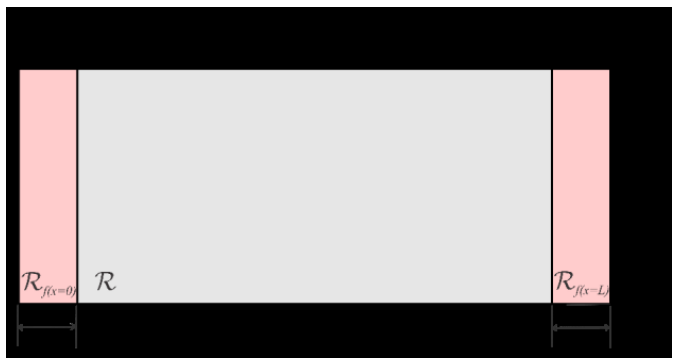

(b)

Fig. C.3. Fixed rectangular plate under tension: (a) geometry and loading; (b) PD model with fictitious regions.

These boundary conditions along $x=0$ with $x_{f} \in R_{f(x=0)}$ are enforced as

$$
\begin{aligned}
& u_{f}\left(x_{f}, y_{f}, t+\Delta t\right)=-u(x, y, t), \\
& v_{f}\left(x_{f}, y_{f}, t+\Delta t\right)=-v(x, y, t) .
\end{aligned}
$$

Along $x=L$ with $x_{f} \in R_{f(x=L)}$, the applied nonzero normal and zero shear stresses are enforced as

$$
\begin{aligned}
& u_{f}\left(x_{f}, y_{f}, t+\Delta t\right)=\left[\frac{\sigma_{o}\left(1-v^{2}\right)}{E}-v \frac{v\left(x, y^{+}, t\right)-v\left(x, y^{-}, t\right)}{y^{+}-y^{-}}\right]\left(x_{f}-x\right)+u(x, y, t), \\
& v_{f}\left(x_{f}, y_{f}, t+\Delta t\right)=\left[-\frac{u\left(x, y^{+}, t\right)-u\left(x, y^{-}, t\right)}{y^{+}-y^{-}}\right]\left(x_{f}-x\right)+v(x, y, t) .
\end{aligned}
$$

The PD predictions successfully capture the non-uniform variation of horizontal and vertical displacements near the fixed boundary and corners as shown in Fig.C.4. These results confirm the validity of the implementation for enforcing displacement constraints as well as traction boundary conditions.

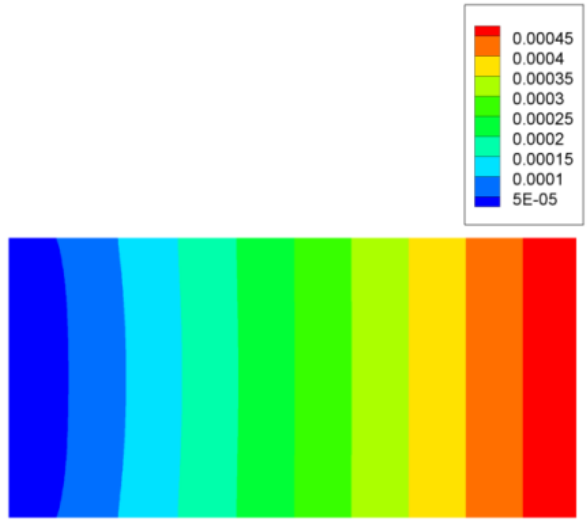

a)

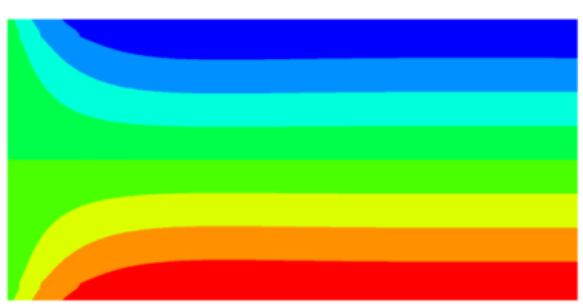

(b)

Fig. C.4 PD solutions for a) Horizontal displacement b) Vertical displacement 